\title{
The homology of spaces of simple topological measures
}

by

\author{
Ø. Johansen and A. B. Rustad (Trondheim)
}

\begin{abstract}
The simple topological measures $X^{*}$ on a q-space $X$ are shown to be a superextension of $X$. Properties inherited from superextensions to topological measures are presented. The homology groups of various subsets of $X^{*}$ are calculated. For a qspace $X, X^{*}$ is shown to be a q-space. The homology of $X^{*}$ when $X$ is the annulus is calculated. The homology of $X^{*}$ when $X$ is a more general genus one space is investigated. In particular, $X^{*}$ for the torus is shown to have a retract homeomorphic to an infinite product of circles.
\end{abstract}

1. Introduction. Throughout the text we will assume that $X$ (and $Y$ ) is a compact Hausdorff space. We will let $\mathcal{O}(X)$ and $\mathcal{C}(X)$ denote the open and closed subsets of $X$ respectively. Furthermore we put $\mathcal{A}(X)=\mathcal{O}(X) \cup$ $\mathcal{C}(X)$. When there is no confusion concerning the space in question, we will omit the space from the notation. A positive set function $\mu: \mathcal{A}(X) \rightarrow \mathbb{R}^{+}$ is a topological measure if:

(i) $\mu\left(\biguplus_{i=1}^{n} A_{i}\right)=\sum_{i=1}^{n} \mu A_{i}$ (where $\biguplus$ indicates disjoint union, and all $A_{i}$ and $\bigcup A_{i}$ are assumed to be in $\mathcal{A}$ ).

(ii) $\mu U=\sup \{\mu C: C \subset U, C \in \mathcal{C}\}$ for all $U \in \mathcal{O}$.

We denote the set of all topological measures on $X$ by $Q(X)$. The $\{0,1\}$ valued topological measures are referred to as simple topological measures and denoted by $X^{*}$. Note that the simple topological measures are not generally all the extreme topological measures.

The topological measures originated in [1], and were formerly called quasi-measures. It was proved in [9] that topological measures are countably additive. Their definition only differs from that of regular Borel measures by their domain of definition. However, they constitute a vastly larger class of set functions with a rich mathematical structure, the most distinctive difference being that topological measures need not be subadditive.

Homological techniques have become of considerable importance for topological measures (cf. [11], [3] and [7]). We continue this work here with

2000 Mathematics Subject Classification: 28Cxx, 46M18, 54Uxx. 
the Čech homology functor as our main vehicle. A remarkable correspondence between superextensions and simple topological measures is presented yielding several results about simple topological measures.

Next we turn to the homology of various subsets of $X^{*}$. In particular, the homology of the sets $C^{*}=\left\{\mu \in X^{*}: \mu C=1\right\}$ for $C \in \mathcal{C}(X)$ (where $X$ is a q-space) is calculated. It is shown that $X^{*}$ is a q-space (see Section 2 for definition) whenever $X$ is a q-space. Solid maps are shown to be monotone in general only when $X$ is a q-space.

Then we turn to more general spaces. Homotopies on $X$ are lifted to $X^{*}$. The homology of the space of simple topological measures on the annulus is shown to be isomorphic to the homology of the circle. Image transformations are constructed from solid sets on spaces with genus one, enabling the calculation of homology groups on such spaces. In particular, the collection of simple topological measures on the torus has a retract homeomorphic to a countable product where each factor has a circle as a retract. The latter reveals the complexity of the homology of the topological measures on the torus (as opposed to the annulus).

\section{Basic results}

2.1. Topological measures. The family of normalized topological measures is a convex set. Its extreme points are, however, much more complex than the Dirac measures. They are not in general $\{0,1\}$-valued.

Definition 1. The space of representable topological measures is the convex closure of the set of simple topological measures, and will be denoted by $Q_{\mathrm{r}}(X)$.

Notation 2. We will denote the Borel probability measures on $X$ by $P(X)$. Its extreme points, the Dirac measures, will be denoted by $P_{\mathrm{e}}(X)$. Moreover, the normalized topological measures on $X$ (i.e. the topological measures with $\mu(X)=1$ ) will be denoted by $Q_{1}(X)$.

The integral of $f \in C(X)$ with respect to a topological measure $\mu$ in $X$ is defined as follows: Let $\mu_{f}$ denote the topological measure given by $\mu_{f}(A)=\mu\left(f^{-1}(A)\right)$ for $A \in \mathcal{A}(X)$. Then $\mu_{f}$ extends uniquely to a Borel measure on $\mathbb{R}$ (all topological measures on one-dimensional spaces extend uniquely to regular Borel measures, cf. [14]). Hence we have the definition

$$
\mu(f)=\int_{\operatorname{sp} f} x d \mu_{f}(x) \quad \text { where } \quad \operatorname{sp} f=\{f(x): x \in X\} .
$$

The integrals (with respect to topological measures), and hence topological measures, were shown in [1] to be uniformly continuous. That is, for $\mu \in$ $Q(X)$ we have $\|\mu\|=\mu(X)$, and $|\mu(f)-\mu(g)| \leq\|\mu\|\|f-g\|_{\infty}$ for all $f, g \in C(X)$. In [2] a weak topology in $Q_{1}(X)$ was introduced: Any function 
$f \in C(X)$ may be represented as a functional $\widehat{f}$ on $Q(X)$ by $\widehat{f}(\mu)=\mu(f)$, where the map $f \mapsto \widehat{f}$ is a non-linear Gelfand transform. The topology on $Q(X)$ is defined to be the topology induced by the separating space of functionals $\{\widehat{f}: f \in C(X)\}$. This turns $Q_{1}(X), Q_{\mathrm{r}}(X)$ and $X^{*}$ into compact Hausdorff spaces. Moreover, the sets $V^{*}=\left\{\mu \in X^{*}: \mu(V)=1\right\}(V \in \mathcal{O}(X))$ form a subbase for the topology of $X^{*}$.

For the remainder of this subsection our spaces will be assumed connected and locally connected.

Definition 3. If a set $A \in \mathcal{A}$ and its complement are both connected we will call the set solid. A restriction to solid sets will be denoted with a subscript $\mathrm{s}$ (e.g. $\mathcal{C}_{\mathrm{S}}$ will denote the compact solid sets). We will let the index $\mathrm{c}$ indicate the restriction to connected sets, e.g. $\mathcal{C}_{\mathrm{c}}(X)$ denotes the closed, connected subsets of $X$.

We recall the following two results from [3].

Proposition 4. Let $K \in \mathcal{C}_{\mathrm{s}}, U \in \mathcal{O}$ and $K \subset U$. Then there is a set $V \in \mathcal{O}_{\mathrm{s}}$ such that $K \subset V \subset \bar{V} \subset U$.

Proposition 5. If $A \in \mathcal{A}(X)$ is connected then the components of $X \backslash A$ are solid.

It was shown in [8] that $\left\{V^{*}: V \in \mathcal{O}_{\mathrm{s}}(X)\right\}$ is actually a subbase for the topology of $X^{*}$. The solid sets play an important role in the theory of topological measures. For their full use some restrictions on the space $X$ are needed:

Definition 6. A partition $\mathcal{P}=\left\{C_{i}\right\}_{i=1}^{n} \cup\left\{U_{j}\right\}_{j=1}^{m}\left(\emptyset \neq C_{i} \in \mathcal{C}_{\mathrm{s}}(X)\right.$, $\left.i=1, \ldots, n ; \emptyset \neq U_{j} \in \mathcal{C}_{\mathrm{s}}(X), j=1, \ldots, m ; m, n \geq 2 ; X=\biguplus \mathcal{P}\right)$ of $X$ into solid sets is called irreducible if for any proper subset $\mathcal{F} \varsubsetneqq\left\{C_{i}\right\}_{i=1}^{n}, X \backslash \biguplus \mathcal{F}$ is connected. By the genus of $X$ we will mean the supremum of $m-1$ for all irreducible partitions of $X$, or zero if no such partitions exist.

REMARK 7. The genus requirement was treated in [3] and [11]. If $X$ has genus zero, then $X$ can at most be the disjoint union of two solid sets. This property is shared by a large class of spaces (e.g. when $X$ is simply connected). Our characterization of genus through finite unions of solid sets is due to [7], where it is shown that if the genus is larger than zero, then it is always possible to find an irreducible partition of $X$ into solid sets such that $n=2$ and $m-1$ is the genus of $X$.

Definition 8. A connected, locally connected, compact Hausdorff space with genus zero is called a $q$-space.

The solid sets constitute a small and manageable family of sets that totally determines a topological measure on a locally connected continuum. This is illustrated by the solid set functions, introduced in [3]. In particular 
they are invaluable tools for constructing topological measures. We include their definition and an extension theorem:

DeFinition 9. Let $X$ be a locally connected continuum. Then a function $\mu: \mathcal{A}_{\mathrm{s}} \rightarrow \mathbb{R}^{+}$is a solid set function if it satisfies:

(i) $\sum_{i=1}^{n} \mu C_{i} \leq \mu C$ whenever $\biguplus_{i=1}^{n} C_{i} \subset C$ with $C_{i}, C \in \mathcal{C}_{\mathrm{s}}$ for $i=$ $1, \ldots, n$.

(ii) $\mu U=\sup \left\{\mu C: C \subset U, C \in \mathcal{C}_{\mathrm{s}}\right\}$ for all $U \in \mathcal{O}_{\mathrm{s}}$.

(iii) $\mu A+\mu(X \backslash A)=\mu X$.

(iv) $\sum_{A \in \mathcal{P}} \mu(A)=\mu X$ for any irreducible partition $\mathcal{P}$ of $X$.

REMARK 10. Note that q-spaces have no irreducible partitions, so the fourth requirement is redundant for q-spaces.

THEOREM 11. If $X$ is a locally connected continuum and $\mu$ is a solid set function, then $\mu$ extends uniquely to a topological measure on $X$. Conversely, the restriction of a topological measure on $X$ to the solid sets is a solid set function.

Image transformations were introduced in [4] as natural topological measure preserving transformations. We include their definition and construction from solid sets:

Definition 12. Let $X$ and $Y$ be compact Hausdorff spaces. An image transformation is a map $q: \mathcal{A}(X) \rightarrow \mathcal{A}(Y)$ with $q(\mathcal{O}(X)) \subset \mathcal{O}(Y)$ satisfying:

(a) $A \cap B=\emptyset \Rightarrow q A \uplus q B=q(A \cup B)$.

(b) $U_{\lambda} \nearrow U \Rightarrow q U_{\lambda} \nearrow q U$ whenever $U_{\lambda}, U \in \mathcal{O}(X)$ and $\lambda \in \Lambda$ where $\Lambda$ is a directed set.

(c) $q X=Y$.

The adjoint of an image transformation $q: \mathcal{A}(X) \rightarrow \mathcal{A}(Y)$ is the map $q^{*}: Q(Y) \rightarrow Q(X)$ defined by

$$
q^{*} \mu(A)=\mu(q A), \quad \mu \in Q(Y), A \in \mathcal{A}(X) .
$$

Moreover, the map $q^{*}: Q(Y) \rightarrow Q(X)$ is continuous.

Proposition 13. Let $X$ be a q-space. A map $q: \mathcal{A}_{\mathrm{s}}(X) \rightarrow \mathcal{A}(Y)$ extends uniquely to an image transformation $\widetilde{q}: \mathcal{A}(X) \rightarrow \mathcal{A}(Y)$ if and only if the following hold:

(a') If $A, A_{i} \in \mathcal{A}_{\mathrm{s}}$ for $i=1, \ldots, n$ and $\biguplus A_{i} \subset A$, then $\biguplus q\left(A_{i}\right) \subset q A$.

$\left(\mathrm{b}^{\prime}\right)$ If $U, U_{\lambda} \in \mathcal{O}_{\mathrm{s}}$ and $U_{\lambda} \nearrow U$ with $\lambda \in \Lambda$ where $\Lambda$ is a directed set, then $q U_{\lambda} \nearrow q U$.

$\left(\mathrm{c}^{\prime}\right)$ For any $A \in \mathcal{A}_{\mathrm{s}}$ we have $q(A) \uplus q(X \backslash A)=Y$.

We have the following definition and proposition from [13]: 
Definition 14. An image transformation $q: \mathcal{A}(X) \rightarrow \mathcal{A}(Y)$ will be called a solid image transformation if $q\left(\mathcal{A}_{\mathrm{s}}(X)\right) \subset \mathcal{A}_{\mathrm{s}}(Y)$. A continuous function is called a solid function if its inverse image is a solid image transformation.

Proposition 15 (Urysohn's lemma for solid variables). Let $X$ be any connected and locally connected compact Hausdorff space. If $C \in \mathcal{C}_{\mathrm{s}}(X)$ and $F \in \mathcal{C}(X)$ are disjoint and non-empty, there is a solid function $f: X \rightarrow[0,1]$ such that $\left.f\right|_{C} \equiv 0$ and $\left.f\right|_{F} \equiv 1$. If in addition $X$ is a metric space we may assume that $f^{-1}(0)=C$.

2.2. Superextensions. Superextensions of topological spaces are constructed similarly to Wallman compactifications, with filters replaced by linked systems. A comprehensive presentation of the subject and its achievements may be found in [12].

Definition 16. Let $\mathcal{S}$ be a closed subbase of $X$. A linked system in $\mathcal{S}$ is a collection $\mathcal{M} \subset \mathcal{S}$ such that any two members of $\mathcal{M}$ intersect. A maximal linked system in $\mathcal{S}$ is a linked system not properly contained in any other linked system in $\mathcal{S}$.

Definition 17. The superextension of $X$ relative to $\mathcal{S}, \lambda(X, \mathcal{S})$, is the topological space defined as the set of all maximal linked systems in $\mathcal{S}$ with the closed subbase

$$
\mathcal{S}^{*}=\left\{C^{*}: C \in \mathcal{S}\right\}
$$

where $C^{*}$ denotes the set of all maximal linked systems in $\mathcal{S}$ containing $C$.

Definition 18. A topological space $X$ is said to be supercompact if there is a closed subbase $\mathcal{S}$ of $X$ such that each linked system $\mathcal{M}$ in $\mathcal{S}$ satisfies

$$
\bigcap\{M: M \in \mathcal{M}\} \neq \emptyset
$$

Such subbases are called binary.

Definition 19. A closed subbase $\mathcal{S}$ of $X$ is called a $T_{1}$-subbase if for each $S \in \mathcal{S}$ and for each $x \in X-S$ there exists a $T \in \mathcal{S}$ such that $x \in T$ and $T \cap S=\emptyset$. A subbase $\mathcal{S}$ of $X$ is called normal if for each disjoint pair $S_{1}, S_{2} \in \mathcal{S}$ there are $S_{1}^{\prime}, S_{2}^{\prime} \in \mathcal{S}$ such that $S_{1} \subset X \backslash S_{1}^{\prime}, S_{2} \subset X \backslash S_{2}^{\prime}$, and $S_{1}^{\prime} \cup S_{2}^{\prime}=X$.

It should be noted that supercompact spaces are compact, and that a superextension is supercompact with $\mathcal{S}^{*}$ as a binary $T_{1}$-subbase. Moreover, if $\mathcal{S}$ is normal, then $\mathcal{S}^{*}$ is normal.

We include the following result from [12]:

TheOREM 20. Let $X$ be a connected topological space. If $X$ admits a normal $T_{1}$-subbase $\mathcal{S}$, then $\lambda(X, \mathcal{S})$ is connected and locally connected, acyclic, 
and a Lefschetz space. Moreover, if $X$ is a metric continuum then $\lambda(X, \mathcal{S})$ is an absolute retract, in particular it is contractible.

3. Correspondence between simple topological measures and superextensions. Throughout this section we will assume $X$ to be a qspace. The closed solid sets constitute a normal closed $T_{1}$-subbase of $X$ by a straightforward application of Proposition 4. We have the following surprising correspondence:

TheOREM 21. There is a homeomorphism $\phi: X^{*} \rightarrow \lambda\left(X, \mathcal{C}_{\mathrm{s}}\right)$ given by

$$
\phi(\mu)=\left\{C \in \mathcal{C}_{\mathrm{s}}: \mu(C)=1\right\},
$$

where $X^{*}$ is endowed with the weak* topology.

Proof. Let $\mu \in X^{*}$, and let $C_{1}, C_{2} \in \phi(\mu)$. Then $\mu\left(C_{1}\right)=\mu\left(C_{2}\right)=1$, so $C_{1} \cap C_{2} \neq \emptyset$. Thus $\phi(\mu)$ is a linked system. If $C \notin \phi(\mu), C \in \mathcal{C}_{\mathrm{s}}$, then $\mu\left(C^{\mathrm{c}}\right)=1$, hence by regularity of $\mu$ there is $C^{\prime} \in \mathcal{C}_{\mathrm{s}}$ with $C^{\prime} \cap C=\emptyset$ and $\mu\left(C^{\prime}\right)=1$, so $C \cup \phi(\mu)$ is not linked. Thus $\phi(\mu)$ is a maximal linked system. Since $\phi(\mu)$ determines the value of $\mu$ on solid sets, $\phi$ is one-to-one.

Conversely, let $S \in \lambda\left(X, \mathcal{C}_{\mathrm{s}}\right)$. Define $\mu: \mathcal{C}_{\mathrm{s}} \rightarrow\{0,1\}$ by $\mu(C)=1$ if and only if $C \in S$, and extend $\mu$ to $\mathcal{A}_{\mathrm{s}}$ by $\mu(O)=1-\mu\left(O^{\mathrm{c}}\right)$ for $O \in \mathcal{O}_{\mathrm{s}}$. Then $\mu$ fulfills requirement 9 (iii) by definition. For 9(i) at most one of the sets $C_{i}, i=1, \ldots, n$, can be in $S$ since they are disjoint. Now $C$ contains each of them and hence must also be in $S$, implying 9(i). If none of the sets $C_{i}$, $i=1, \ldots, n$, are in $S$ then 9(i) is trivially fulfilled. It remains to prove the regularity 9(ii), so let $O \in \mathcal{O}_{\mathrm{s}}$ be arbitrary. If $\mu O=0$ then any compact set $C \subset O$ is disjoint from $O^{\mathrm{c}} \in S$, and hence $\mu O=\sup \{\mu C: C \subset O$, $\left.C \in \mathcal{C}_{\mathrm{s}}\right\}=0$. If $\mu O=1$ then $O^{\mathrm{c}} \notin S$, hence there must be some $C \in S$ with $C \cap O^{c}=\emptyset$ by the maximality of $S$. Accordingly $C \subset O$ with $\mu C=1$, so $\mu O=\sup \left\{\mu C: C \subset O, C \in \mathcal{C}_{\mathrm{s}}\right\}=1$.

Finally, the continuity of $\phi$ and $\phi^{-1}$ is evident by considering closed subbases.

REMARK 22. The correspondence between maximal linked systems and simple topological measures on q-spaces was already noted in [11], but the connection to the topological construct of a superextension was not made.

EXAMPle 23. The image transformation $\Psi: X \rightarrow X^{*}$ defined by $\Psi(A)$ $=A^{*}$ where $A^{*}=\left\{\mu \in X^{*}: \mu(A)=1\right\}$ was presented in [4]. It is noteworthy that this image transformation is, through the correspondence in Theorem 21 , analogous to the $*$-operation of Definition 17 . We will return to this image transformation after establishing some immediate consequences of Theorem 21.

Corollary 24. If $X$ is a metric q-space, then $X^{*}$ is an absolute retract, in particular it is contractible. 
Corollary 25. If $X$ is a q-space, then:

(i) $X^{*}$ is connected and locally connected.

(ii) $X^{*}$ is acyclic.

(iii) $X^{*}$ is a Lefschetz space.

REMARK 26. The connectedness of $X^{*}$ was proven in [11], and the local connectedness in [10], so those results are not new. The notion of a Lefschetz space may be found in [5], and refers to a conditional fixed point property of selfmaps of $X^{*}$. Acyclicity is meant with respect to the Cech homology functor, $H=\left(H_{n}\right)_{n \in \mathbb{N}}$, over a field $\mathbb{F}$; we refer the reader to [6] for a good source of information on homology theory. Since $X^{*}$ is acyclic, the Lefschetz property implies that every continuous map of $X^{*}$ into itself has a fixed point.

ExAMPLE 27. Let $q: \mathcal{A}(X) \rightarrow \mathcal{A}(X)$ be an image transformation. The adjoint map $q^{*}: X^{*} \rightarrow X^{*}$ given by $\left(q^{*} \mu\right)(A)=\mu(q(A))$ for $A \in \mathcal{A}(X)$ and $\mu \in Q(X)$ was shown in [4] to be continuous. By Corollary 25(iii), $q^{*}$ must have a fixed point in $X^{*}$.

4. Homological results for simple topological measures on qspaces. Image transformations were introduced in [4] as natural continuous topological measure preserving transformations. Hence they are natural tools to consider when calculating the various homology groups of spaces of simple topological measures. We start by introducing a fundamental class of image transformations to achieve this. Again we will assume $X$ to be a q-space throughout this section.

Lemma 28. For $C \in \mathcal{C}(X)$ define a set map $q_{C}: \mathcal{A}_{\mathrm{s}}(X) \rightarrow \mathcal{A}(X)$ by

$$
q_{C}(A)= \begin{cases}\emptyset & \text { if } C \cap A=\emptyset, \\ A & \text { if } \emptyset \neq C \cap A \neq C, A \in \mathcal{A}_{\mathrm{s}}(X), \\ X & \text { if } C \subset A .\end{cases}
$$

Then $q_{C}$ extends uniquely to an image transformation. Moreover, if $C_{1} \cap C_{2}$ $\neq \emptyset$ and $C_{1}, C_{2} \in \mathcal{C}(X)$, then $q_{C_{1}} \circ q_{C_{2}}=q_{C_{2}} \circ q_{C_{1}}$.

Proof. Requirement $\left(\mathrm{c}^{\prime}\right)$ of Proposition 13 is trivially fulfilled by extension. Requirement $\left(\mathrm{a}^{\prime}\right)$ is a straightforward verification for the set map $q_{C}$. For the regularity requirement $\left(b^{\prime}\right)$, we have three cases to consider for a net $U_{\lambda} \nearrow U$ of open solid sets. The case $q_{C} U=\emptyset$ implies $q_{C} U_{\lambda}=\emptyset$ for all $\lambda \in \Lambda$. The next two cases, $q_{C} U=U$ and $q_{C} U=X$, are handled by a standard compactness argument. Hence $q_{C}$ extends uniquely to an image transformation. If $A \in \mathcal{A}_{\mathrm{s}}(X)$, then the only way in which $\left(q_{C_{1}} \circ q_{C_{2}}\right)(A)$ can be different from $\left(q_{C_{2}} \circ q_{C_{1}}\right)(A)$ is if one of $q_{C_{1}}(A)$ and $q_{C_{2}}(A)$ is $\emptyset$ and the other is $X$; but this is impossible if $C_{1}$ and $C_{2}$ intersect. 
Since topological measures are inner regular it is an easy exercise to show that additivity on disjoint open sets holds in general. We have the following:

Theorem 29. Let $C \in \mathcal{C}_{\mathrm{c}}(X)$. Then $q_{C}^{*}$ is a retraction of $X^{*}$ onto $C^{*}$. Moreover, if $C_{1}, \ldots, C_{n} \in \mathcal{C}_{\mathrm{c}}(X)$ are pairwise intersecting, then $q_{C_{1}}^{*} \circ \ldots \circ q_{C_{n}}^{*}$ is a retraction of $X^{*}$ onto $C_{1}^{*} \cap \ldots \cap C_{n}^{*}$.

Proof. Let $\mu \in X^{*}$. Then

$$
q_{C}^{*}(\mu)(C)=1-\sum_{\lambda \in \Lambda} \mu\left(q\left(U_{\lambda}\right)\right)=1-\sum_{\lambda \in \Lambda} \mu(\emptyset)=1,
$$

where $\left\{U_{\lambda}\right\}_{\lambda \in \Lambda}$ are the (solid and open) components of $X \backslash C$. Thus $q_{C}^{*}(\mu)$ $\in C^{*}$. Assume further that $\mu \in C^{*}$ and $A \in \mathcal{A}_{\mathrm{s}}(X)$. Then in each of the three cases in the definition of $q_{C}, \mu\left(q_{C}(A)\right)=\mu(A)$, hence $q_{C}^{*}(\mu)=\mu$. Accordingly, $q_{C}^{*}$ is a retraction of $X^{*}$ onto $C^{*}$.

Clearly, $\left(q_{C_{1}}^{*} \circ \ldots \circ q_{C_{n}}^{*}\right)\left(X^{*}\right) \subset C_{1}^{*}$ and by commutativity it follows that $\left(q_{C_{1}}^{*} \circ \ldots \circ q_{C_{n}}^{*}\right)\left(X^{*}\right) \subset C_{1}^{*} \cap \ldots \cap C_{n}^{*}$. If $\mu \in C_{1}^{*} \cap \ldots \cap C_{n}^{*}$, it is immediate that $\left(q_{C_{1}}^{*} \circ \ldots \circ q_{C_{n}}^{*}\right)(\mu)=\mu$.

Corollary 30. If $C \in \mathcal{C}_{\mathrm{c}}(X)$, then $C^{*}$ is acyclic. Moreover, if $C_{1}, \ldots, C_{n}$ $\in \mathcal{C}_{\mathrm{c}}(X)$ are pairwise intersecting, then $C_{1}^{*} \cap \ldots \cap C_{n}^{*}$ is acyclic. In particular, $C_{1}^{*} \cap \ldots \cap C_{n}^{*}$ is non-empty.

Proof. Acyclicity follows since retracts of acyclic spaces are acyclic. The set $C_{1}^{*} \cap \ldots \cap C_{n}^{*}$ is non-empty since it is the image of the map $q_{C_{1}}^{*} \circ \ldots \circ q_{C_{n}}^{*}$.

Corollary 31. Let $C_{1}, \ldots, C_{n} \in \mathcal{C}_{\mathrm{c}}(X)$ and let $F_{i}=\bigcap_{j \in I_{i}} C_{j}^{*}$, where $I_{i} \subset\{1, \ldots, n\}, i=1, \ldots, m$. If $F_{1}, \ldots, F_{m}$ are pairwise intersecting, then $F_{1} \cap \ldots \cap F_{m} \neq \emptyset$.

Proof. If $F_{1}, \ldots, F_{m}$ are pairwise intersecting, then $\left\{C_{j}: j \in \bigcup_{i=1}^{m} I_{i}\right\}$ must also intersect pairwise.

Corollary 32. If $A \in \mathcal{A}(X)$ is connected, then $A^{*}$ is connected.

Proof. For $C \in \mathcal{C}_{\mathrm{c}}(X)$ we have $q_{C}^{*}\left(X^{*}\right)=C^{*}$, implying connectedness of $C^{*}$. For $O \in \mathcal{O}_{\mathrm{c}}(X), O^{*}=\bigcup\left\{C^{*}: C \in \mathcal{C}_{\mathrm{c}}(X), C \subset O\right\}$ and the union is directed, hence $O^{*}$ is also connected.

Recall that a map between compact Hausdorff spaces is called monotone if inverse images of connected sets are connected. We have the following definition for image transformations:

Definition 33. An image transformation $q: \mathcal{A}(X) \rightarrow \mathcal{A}(Y)$ is called monotone if $q\left(\mathcal{A}_{\mathrm{c}}(X)\right) \subset \mathcal{A}_{\mathrm{c}}(Y)$.

Note that any monotone image transformation is necessarily solid since image transformations preserve complements. This, of course, also applies to functions. 
Corollary 34. The image transformation $\Psi: \mathcal{A}(X) \rightarrow \mathcal{A}\left(X^{*}\right)$ is monotone.

The Cech homology functor is continuous in the sense that to limits of inverse systems of sets correspond inverse limits of the corresponding homology groups. We obtain the following result:

Proposition 35. Let $C \in \mathcal{C}(X)$. Then $H_{n}\left(C^{*}\right)=0$ for all $n \neq 0$, and $H_{0}\left(C^{*}\right) \cong H_{0}(C)$.

Proof. If $C \in \mathcal{C}(X)$ has finitely many components, then the result follows from Corollary 30 by taking the sum of the homologies of each component. For any $C$, let $\left\{C_{\lambda}\right\}_{\lambda \in \Lambda}$ be the family of compact sets with finitely many components containing $C$, ordered by reverse inclusion. Then $\left\{C_{\lambda}^{*}\right\}_{\lambda \in \Lambda}$ together with the inclusion mappings is an inverse system with $C^{*}$ as its intersection, and so by the continuity of the Čech homology, $H_{n}\left(C^{*}\right)=\lim H_{n}\left(C_{\lambda}^{*}\right)$. For $n \neq 0$ this gives the result. For $n=0$ we have $H_{0}\left(C_{\lambda}^{*}\right) \cong H_{0}\left(C_{\lambda}\right)$ as inverse systems, so $H_{0}\left(C^{*}\right)=\lim _{\longleftarrow} H_{0}\left(C_{\lambda}^{*}\right)=\lim _{\longleftarrow} H_{0}\left(C_{\lambda}\right)=H_{0}(C)$.

The Čech homology sequence is exact for compact sets, and hence the Mayer-Vietoris sequence is exact for compact sets. We have the following useful result:

Proposition 36. If $C_{1}, \ldots, C_{n} \in \mathcal{C}_{\mathrm{c}}(X)$ are pairwise intersecting, then any combination of unions and intersections of the sets $C_{1}^{*}, \ldots, C_{n}^{*}$ is acyclic.

Proof. We may assume that any combination of unions and intersections is of the form

$$
\bigcup_{j=1}^{m} \bigcap_{k=1}^{n_{j}} C_{j, k}^{*}, \quad C_{j, k} \in\left\{C_{1}, \ldots, C_{n}\right\} .
$$

Now $\bigcap_{k=1}^{n_{j}} C_{j, k}^{*}$ is acyclic for each $j$. This shows the result for $m=1$. We proceed by induction on $m$. Assume the assertion to be true for some $m \geq 1$. We obtain the Mayer-Vietoris sequence

$$
\begin{aligned}
0=\widetilde{H}_{n}\left(\bigcup_{j=1}^{m} \bigcap_{k=1}^{n_{j}} C_{j, k}^{*}\right) \oplus \widetilde{H}_{n} & \left(\bigcap_{k=1}^{n_{m+1}} C_{m+1, k}^{*}\right) \rightarrow \widetilde{H}_{n}\left(\bigcup_{j=1}^{m+1} \bigcap_{k=1}^{n_{j}} C_{j, k}^{*}\right) \\
& \rightarrow \widetilde{H}_{n-1}\left(\left(\bigcup_{j=1}^{m} \bigcap_{k=1}^{n_{j}} C_{j, k}^{*}\right) \cap\left(\bigcap_{k=1}^{n_{m+1}} C_{m+1, k}^{*}\right)\right) .
\end{aligned}
$$

The acyclicity of $\bigcup_{j=1}^{m+1} \bigcap_{k=1}^{n_{j}} C_{j, k}^{*}$ follows since

$$
\left(\bigcup_{j=1}^{m} \bigcap_{k=1}^{n_{j}} C_{j, k}^{*}\right) \cap\left(\bigcap_{k=1}^{n_{m+1}} C_{m+1, k}^{*}\right)=\bigcup_{j=1}^{m}\left(\left(\bigcap_{k=1}^{n_{j}} C_{j, k}^{*}\right) \cap\left(\bigcap_{k=1}^{n_{m+1}} C_{m+1, k}^{*}\right)\right)
$$


and by induction

$$
\widetilde{H}_{n-1}\left(\bigcup_{j=1}^{m}\left(\left(\bigcap_{k=1}^{n_{j}} C_{j, k}^{*}\right) \cap\left(\bigcap_{k=1}^{n_{m+1}} C_{m+1, k}^{*}\right)\right)\right)=0 .
$$

Example 37. Consider pairwise intersecting sets $C_{1}, C_{2}, C_{3} \in \mathcal{C}_{\mathrm{c}}(X)$, e.g. the three edges of a triangle. Then by Proposition 36, $C_{1}^{*} \cup C_{2}^{*} \cup C_{3}^{*}$ is acyclic, whereas the set $C_{1} \cup C_{2} \cup C_{3}$ may be homeomorphic to a circle. This is a rather disappointing result, but as the next example illustrates we need at least four sets to obtain more complicated homology of $X^{*}$.

ExAmple 38. Let $C_{1}, \ldots, C_{4} \in \mathcal{C}_{\mathrm{c}}(X)$ be the edges of a square numbered clockwise. Then $\left(C_{1}^{*} \cup C_{2}^{*}\right) \cap\left(C_{3}^{*} \cup C_{4}^{*}\right)=\left(C_{1}^{*} \cap C_{4}^{*}\right) \cup\left(C_{2}^{*} \cap C_{3}^{*}\right)$ where the latter is a disjoint union. Thus we obtain the following Mayer-Vietoris sequence:

$$
\begin{aligned}
0 & =\widetilde{H}_{1}\left(C_{1}^{*} \cup C_{2}^{*}\right) \oplus \widetilde{H}_{1}\left(C_{3}^{*} \cup C_{4}^{*}\right) \rightarrow \widetilde{H}_{1}\left(C_{1}^{*} \cup \ldots \cup C_{4}^{*}\right) \\
& \rightarrow \widetilde{H}_{0}\left(\left(C_{1}^{*} \cap C_{4}^{*}\right) \uplus\left(C_{2}^{*} \cap C_{3}^{*}\right)\right) \rightarrow \widetilde{H}_{0}\left(C_{1}^{*} \cup C_{2}^{*}\right) \oplus \widetilde{H}_{0}\left(C_{3}^{*} \cup C_{4}^{*}\right)=0 .
\end{aligned}
$$

The homology group $\widetilde{H}_{0}\left(\left(C_{1}^{*} \cap C_{4}^{*}\right) \uplus\left(C_{2}^{*} \cap C_{3}^{*}\right)\right)$ is isomorphic to the field $\mathbb{F}$, and hence $\widetilde{H}_{1}\left(C_{1}^{*} \cup \ldots \cup C_{4}^{*}\right) \cong \mathbb{F}$. This illustrates that $C_{1}^{*} \cup \ldots \cup C_{4}^{*}$ has the same first homology group as the circle.

The preceding example may be generalized to $n$-cubes; we include the calculations below:

Example 39. Let $F_{i, s} \in \mathcal{C}_{\mathrm{c}}(X), i=1, \ldots, k, s=0,1$, be such that $F_{i, s} \cap F_{j, t}=\emptyset \Leftrightarrow i=j, s \neq t$ (i.e. the same pairwise intersections as the faces of a $k$-cube). For $I_{0}, I_{1} \subset\{1, \ldots, k\}$ define $F_{I_{0}, I_{1}}=\left(\bigcup_{i \in I_{0}} F_{i, 0}^{*}\right) \cup\left(\bigcup_{i \in I_{1}} F_{i, 1}^{*}\right)$. Then:

(i) $F_{I_{0}, I_{1}}$ is acyclic when $I_{0} \neq I_{1}$.

(ii) $\widetilde{H}_{n}\left(F_{I, I}\right) \cong \widetilde{H}_{n}\left(S^{\operatorname{card} I-1}\right) \cong \begin{cases}0, & n \neq \operatorname{card} I-1, \\ \mathbb{F}, & n=\operatorname{card} I-1 .\end{cases}$

Proof of Example 39. (i) If $I_{0} \cap I_{1}=\emptyset$ then all the sets $\left\{F_{i, 0}, F_{j, 1}\right.$ : $\left.i \in I_{0}, j \in I_{1}\right\}$ intersect pairwise and Proposition 36 applies. Otherwise, let $i \in I_{0} \cap I_{1}$ and put $F=F_{I_{0} \backslash\{i\}, I_{1} \backslash\{i\}}$. Then

$$
F_{I_{0} \backslash\{i\}, I_{1}} \cap F_{I_{0}, I_{1} \backslash\{i\}}=\left(F \cup F_{i, 1}^{*}\right) \cap\left(F \cup F_{i, 0}^{*}\right)=F \cup\left(F_{i, 1}^{*} \cap F_{i, 0}^{*}\right)=F .
$$

We have the Mayer-Vietoris sequence

$0=\widetilde{H}_{n}\left(F_{I_{0} \backslash\{i\}, I_{1}}\right) \oplus \widetilde{H}_{n}\left(F_{I_{0}, I_{1} \backslash\{i\}}\right) \rightarrow \widetilde{H}_{n}\left(F_{I_{0}, I_{1}}\right) \rightarrow \widetilde{H}_{n-1}\left(F_{I_{0} \backslash\{i\}, I_{1} \backslash\{i\}}\right)=0$ and the acyclicity of $F_{I_{0}, I_{1}}$ follows by induction on card $I_{0}+$ card $I_{1}$.

(ii) The statement is clear when card $I \leq 1$. Otherwise let $i \in I$; by (i) we have the Mayer-Vietoris sequence 


$$
\begin{aligned}
0=\widetilde{H}_{n}\left(F_{I \backslash\{i\}, I}\right) \oplus \widetilde{H}_{n}\left(F_{I, I \backslash\{i\}}\right) \rightarrow \widetilde{H}_{n}\left(F_{I, I}\right) & \\
& \rightarrow \widetilde{H}_{n-1}\left(F_{I \backslash\{i\}, I \backslash\{i\}}\right) \rightarrow \widetilde{H}_{n-1}\left(F_{I \backslash\{i\}, I}\right) \oplus \widetilde{H}_{n-1}\left(F_{I, I \backslash\{i\}}\right)=0,
\end{aligned}
$$

yielding the result by induction on card $I$.

5. Conditions for q-spaces. Throughout this section $X$ and $Y$ will be assumed to be locally connected continua. Conditions for $X$ and $X^{*}$ being q-spaces will be given. We start with a characterization of q-spaces in terms of compact sets:

Proposition 40. Let $X$ be a connected, locally connected compact Hausdorff space. If $C_{1}^{\circ} \cup C_{2}^{\circ}=X$ with $C_{1}, C_{2} \in \mathcal{C}_{\mathrm{c}}(X)$ implies $C_{1} \cap C_{2} \in \mathcal{C}_{\mathrm{c}}(X)$, then $X$ is a q-space. Conversely, if $X$ is a q-space, then $C_{1} \cup C_{2}=X$ with $C_{1}, C_{2} \in \mathcal{C}_{\mathrm{c}}(X)$ implies $C_{1} \cap C_{2} \in \mathcal{C}_{\mathrm{c}}(X)$.

Proof. Assume to the contrary that $X$ does not have genus zero according to Definition 6. Then there is a collection of sets $A_{1}, \ldots, A_{n} \in \mathcal{A}_{\mathrm{s}}(X)$ such that $X=\biguplus_{i=1}^{n} A_{i}$ with exactly two of the solid sets closed (see Remark 7), say $A_{1}$ and $A_{2}$. We must have $n \geq 4$ and hence may fix two points $x_{1}, x_{2}$ contained respectively in two different open sets, say $A_{3}$ and $A_{4}$. By Proposition 4 there is an open solid set $O_{1}$ such that $A_{1} \subset O_{1} \subset \bar{O}_{1} \subset$ $X \backslash\left(A_{2} \cup\left\{x_{1}, x_{2}\right\}\right)$. Similarly there is also an open solid set $O_{2}$ such that $A_{2} \subset O_{2} \subset \bar{O}_{2} \subset X \backslash\left(\bar{O}_{1} \cup\left\{x_{1}, x_{2}\right\}\right)$. Now put $C_{i}=X \backslash O_{i}, i=1,2$; then $C_{1}^{\circ} \cup C_{2}^{\circ}=X$ and $C_{1}, C_{2} \in \mathcal{C}_{\mathrm{c}}(X)$. However, we have $x_{1}, x_{2} \in C_{1} \cap C_{2} \subset$ $\biguplus_{i=3}^{n} A_{i}$, in particular, $A_{3}$ and $\biguplus_{i=4}^{n} A_{i}$ separate $C_{1} \cap C_{2}$. We must have $C_{1} \cap C_{2} \notin \mathcal{C}_{\mathrm{c}}(X)$, contradicting the hypothesis of the proposition.

Conversely, let $X$ be a q-space and assume $X=C_{1} \cup C_{2}, C_{1}, C_{2} \in \mathcal{C}_{\mathrm{c}}(X)$. Then $\emptyset \neq C_{1}^{*} \cap C_{2}^{*}=\left(C_{1} \cap C_{2}\right)^{*}$ and hence $\left(C_{1} \cap C_{2}\right)^{*}$ is acyclic, implying that $\left(C_{1} \cap C_{2}\right)^{*}$ is connected. If $\left(C_{1} \cap C_{2}\right)^{*}$ is connected, then $C_{1} \cap C_{2}$ must also be connected by Proposition 35 .

Remark 41. We note that in the case of Cech homology this result is stronger than necessary, since the Mayer-Vietoris sequence is exact for compact pairs regardless of whether their interiors cover the space. For other homologies the stronger result in Proposition 40 may be useful.

The Čech homology group $H_{0}$ has the nice property of describing connectivity whereas singular homology describes pathwise connectivity. That is, for a set $A \in \mathcal{A}(X)$ we have $\widetilde{H}_{0}(A)=0$ if and only if $A$ is connected.

Theorem 42. If $X$ is a q-space, then $X^{*}$ is also a q-space.

Proof. By Corollary 25(i), $X^{*}$ is connected and locally connected. Suppose $C_{1}, C_{2} \in \mathcal{C}_{\mathrm{c}}\left(X^{*}\right)$ with $C_{1} \cup C_{2}=X^{*}$. Applying the acyclicity of $X^{*}$ (Corollary 25(ii)) we have the Mayer-Vietoris sequence

$$
0=\widetilde{H}_{1}\left(X^{*}\right) \rightarrow \widetilde{H}_{0}\left(C_{1} \cap C_{2}\right) \rightarrow \widetilde{H}_{0}\left(C_{1}\right) \oplus \widetilde{H}_{0}\left(C_{2}\right)=0,
$$


since $\widetilde{H}_{0}\left(C_{1}\right)=\widetilde{H}_{0}\left(C_{2}\right)=0$ and hence $\widetilde{H}_{0}\left(C_{1}\right) \oplus \widetilde{H}_{0}\left(C_{2}\right)=0$. It follows from the exactness that $\widetilde{H}_{0}\left(C_{1} \cap C_{2}\right)=0$ and accordingly $C_{1} \cap C_{2} \in \mathcal{C}_{\mathrm{c}}(X)$. Finally $X^{*}$ is a q-space by Proposition 40 .

THEOREM 43. The following are equivalent:

(i) $X$ is a q-space.

(ii) Any solid image transformation $q: \mathcal{A}(Y) \rightarrow \mathcal{A}(X)$ is monotone.

(iii) Any solid function $f \in C(X)$ is monotone.

Proof. For (i) $\Rightarrow$ (ii) assume $X$ to be a q-space, and $q: \mathcal{A}(Y) \rightarrow \mathcal{A}(X)$ to be a solid image transformation.

We will first consider compact sets. Let $C \in \mathcal{C}_{\mathrm{c}}(Y)$ be arbitrary and let $\left\{V_{\lambda}\right\}_{\lambda \in \Lambda}$ denote the open solid components of $Y \backslash C$. By regularity we then have $X \backslash q(C)=\biguplus_{\lambda \in \Lambda} q\left(V_{\lambda}\right)$.

We will show that $X \backslash \biguplus_{\lambda \in F} q\left(V_{\lambda}\right)$ is connected whenever $F \subset \Lambda$ is finite. For card $F=1$ this follows since by assumption each $q\left(V_{\lambda}\right)$ is solid. Assume that $X \backslash \biguplus_{\lambda \in F} q\left(V_{\lambda}\right)$ is connected whenever card $F \leq n$. Let $F=F_{1} \uplus F_{2}$ with card $F=n+1$ and $F_{1}$ and $F_{2}$ both non-empty. We have

$$
\begin{aligned}
X \backslash \biguplus_{\lambda \in F} q\left(V_{\lambda}\right) & =\left(X \backslash \biguplus_{\lambda \in F_{1}} q\left(V_{\lambda}\right)\right) \cap\left(X \backslash \biguplus_{\lambda \in F_{2}} q\left(V_{\lambda}\right)\right), \\
X & =\left(X \backslash \biguplus_{\lambda \in F_{1}} q\left(V_{\lambda}\right)\right) \cup\left(X \backslash \biguplus_{\lambda \in F_{2}} q\left(V_{\lambda}\right)\right),
\end{aligned}
$$

and by the induction hypothesis and Proposition 40, $X \backslash \biguplus_{\lambda \in F} q\left(V_{\lambda}\right)$ is connected. This completes the induction.

We see that $\left\{X \backslash \biguplus_{\lambda \in F} q\left(V_{\lambda}\right): F \subset \Lambda, F\right.$ finite $\}$ is a directed family of connected compact sets ordered by reverse inclusion, and so its intersection $q(C)$ is connected.

Now let $O \in \mathcal{O}_{\mathrm{c}}(Y)$ be arbitrary, and let $x_{1}, x_{2} \in q(O)$. Consider the directed family $\left\{U_{\lambda} \subset O: U_{\lambda} \in \mathcal{O}_{\mathrm{c}}(Y), \bar{U}_{\lambda} \subset O\right\}$, which has union $O$; by regularity of $q$ we have $x_{1}, x_{2} \in q\left(U_{\lambda}\right)$ for some $U_{\lambda}$. Then $\left\{x_{1}, x_{2}\right\} \subset q\left(\bar{U}_{\lambda}\right) \subset$ $q(O)$, where $q\left(\bar{U}_{\lambda}\right)$ is connected by the above, and so any $x_{1}, x_{2} \in q(O)$ must be in the same component of $q(O)$, which must therefore be connected. This establishes the monotonicity of $q$.

The implication (ii) $\Rightarrow$ (iii) is trivial. For (iii) $\Rightarrow$ (i) assume that $X$ is not a q-space. By Remark 7 (cf. [7]) we can find disjoint compact solid sets $C_{1}, C_{2}$ such that $X \backslash\left(C_{1} \uplus C_{2}\right)$ is a union $\biguplus_{i=1}^{n} U_{i}$ with $\emptyset \neq U_{i} \in \mathcal{O}_{\mathrm{s}}(X)$ for $i=1, \ldots, n, n \geq 2$, and by Proposition 15 we have a solid function $f: X \rightarrow[0,1]$ with $f\left(C_{1}\right)=0$ and $f\left(C_{2}\right)=1$. We will show that $f^{-1}((0,1))$ is disconnected. Assume the contrary; then $f^{-1}((0,1))$ must be contained in a single component of $X \backslash\left(C_{1} \uplus C_{2}\right)$, say $U_{1}$. But then $U_{2}$ must be contained entirely in either $f^{-1}(0)$ or $f^{-1}(1)$, and so either $\bar{U}_{2} \cap C_{1}=\emptyset$ or $\bar{U}_{2} \cap C_{2}=\emptyset$, 
say the former, i.e. $C_{1} \subset X \backslash \bar{U}_{2}$. But then $X \backslash C_{2}=U_{2} \uplus\left(X \backslash\left(C_{2} \cup \bar{U}_{2}\right)\right)$, contradicting the solidity of $C_{2}$. We have shown that $f^{-1}((0,1))$ is disconnected and so $f$ is not monotone.

6. Results for general spaces. In this section we will consider some methods for calculating the homology of $X^{*}$ for more general spaces. Accordingly, we will only assume $X, Y$ and $Z$ to be compact Hausdorff spaces.

The transformation theorem for integrals holds for integrals with respect to topological measures, i.e. if $\mu \in Q(X), f \in C(X, Y)$ (continuous functions from $X$ to $Y$ ) and $g \in C(Y)$, then

$$
\mu(g \circ f)=\left(f_{*} \mu\right)(g) .
$$

We have the following:

Proposition 44. Let $f: X \times[0,1] \rightarrow Y$ be a homotopy. Then $f$ lifts naturally to a homotopy $\widetilde{f}: X^{*} \times[0,1] \rightarrow Y^{*}$ defined by $\widetilde{f}(\mu, t)=(f(\cdot, t))_{*}(\mu)$ for $\mu \in X^{*}$ and $t \in[0,1]$.

Proof. Assume $f: X \times[0,1] \rightarrow Y$ to be a homotopy and define $\widetilde{f}$ : $X^{*} \times[0,1] \rightarrow Y^{*}$ by $\tilde{f}(\mu, t)=(f(\cdot, t))_{*}(\mu)$ for $\mu \in X^{*}, t \in[0,1]$. It suffices to show that $\tilde{f}$ is continuous. Let $\left(\mu_{\lambda}, t_{\lambda}\right) \rightarrow(\mu, t)$ with $\mu_{\lambda}, \mu \in X^{*}$ and $t_{\lambda}, t \in[0,1]$ be a convergent net in $X^{*} \times[0,1]$. For any $g \in C(Y)$ we have

$$
\begin{aligned}
& \left|\widetilde{f}\left(\mu_{\lambda}, t_{\lambda}\right)(g)-\tilde{f}(\mu, t)(g)\right|=\left|\left[f\left(\cdot, t_{\lambda}\right)_{*}\left(\mu_{\lambda}\right)\right](g)-\left[f(\cdot, t)_{*}(\mu)\right](g)\right| \\
& \quad \leq\left|\left[f\left(\cdot, t_{\lambda}\right)_{*}\left(\mu_{\lambda}\right)\right](g)-\left[f(\cdot, t)_{*}\left(\mu_{\lambda}\right)\right](g)\right|+\left|\left[f(\cdot, t)_{*}\left(\mu_{\lambda}\right)\right](g)-\left[f(\cdot, t)_{*}(\mu)\right](g)\right| \\
& =\left|\mu_{\lambda}\left[(g \circ f)\left(\cdot, t_{\lambda}\right)\right]-\mu_{\lambda}[(g \circ f)(\cdot, t)]\right|+\left|\mu_{\lambda}[(g \circ f)(\cdot, t)]-\mu[(g \circ f)(\cdot, t)]\right| \\
& \leq\left\|(g \circ f)\left(\cdot, t_{\lambda}\right)-(g \circ f)(\cdot, t)\right\|_{\infty}\left\|\mu_{\lambda}\right\|+\left|\mu_{\lambda}[(g \circ f)(\cdot, t)]-\mu[(g \circ f)(\cdot, t)]\right| .
\end{aligned}
$$

The continuity of $\tilde{f}$ now follows from the uniform convergence of $(g \circ f)\left(\cdot, t_{\lambda}\right)$ and the definition of the topology on $X^{*}$.

REMARK 45. It should be noted at this point that the adjoint operation is also a functor when ordinary measures are replaced by $X^{*}$, i.e. $(f \circ g)_{*}=$ $f_{*} \circ g_{*}$ for $g \in C(X, Y), f \in C(Y, Z)$. A comprehensive treatment of the adjoint operation may be found in [4]. The proof of Proposition 44 goes through unchanged with $X^{*}$ and $Y^{*}$ replaced by $Q(X)$ and $Q(Y)$, but we do not need the more general result here.

If $C \in \mathcal{C}(X)$ and $A \in \mathcal{A}(C)$, let $A_{C}^{*}$ denote the zero-one-valued topological measures $\mu$ in $C$ with $\mu A=1$.

Proposition 46. Let $C \in \mathcal{C}(X)$ be a deformation retract of $X$. Then $i_{*}\left(C_{C}^{*}\right)$ is a deformation retract of $X^{*}$, where $i_{*}$ is the adjoint of the inclusion map $i: C \hookrightarrow X$. In particular, $X^{*}$ is homotopically equivalent to $C_{C}^{*}$. 
Proof. There is a homotopy $f: X \times[0,1] \rightarrow X$ and a retraction $r: X \rightarrow C$ such that $f(x, 0)=x$ and $f(x, 1)=(i \circ r)(x)$ for $x \in X$. By Proposition 44 we obtain a homotopy $\widetilde{f}: X^{*} \times[0,1] \rightarrow X^{*}$ with $\widetilde{f}(\mu, 0)=\mu$ and $\widetilde{f}(\mu, 1)$ $=(i \circ r)_{*}(\mu)=\left(i_{*} \circ r_{*}\right)(\mu)$. For $i_{*} \mu \in i_{*}\left(C_{C}^{*}\right)$ we have $\left(i_{*} \circ r_{*}\right)\left(i_{*} \mu\right)=$ $\left(i_{*} \circ\left(r_{*} \circ i_{*}\right)\right)(\mu)=i_{*} \mu$ since $r \circ i$ is the identity map on $C$. Hence $i_{*} \circ r_{*}$ is the identity map on $i_{*}\left(C_{C}^{*}\right)$, establishing that $i_{*}\left(C_{C}^{*}\right)$ is a deformation retract of $X^{*}$. Now, $r \circ i=\operatorname{id}_{C}$ implies that $r_{*} \circ i_{*}=\operatorname{id}_{C^{*}}$ and accordingly $i_{*}\left(C_{C}^{*}\right)$ is homeomorphic to $C_{C}^{*}$, which proves that $X^{*}$ is homotopically equivalent to $C_{C}^{*}$.

Corollary 47. Let $X$ be the annulus. Then $X^{*}$ is homotopically equivalent to the circle. In particular, the homology of $X^{*}$ is isomorphic to that of the circle.

Proof. The circle $\mathbb{T}$ is a deformation retract of the annulus, so Proposition 46 applies. The circle is one-dimensional and hence all topological measures extend to regular Borel measures (cf. [14]). Accordingly $\mathbb{T}^{*}$ is homeomorphic to the circle, establishing the assertion.

The homology of the simple topological measures on the torus $\mathbb{T}^{2}$ has been a bit of a mystery. The next result will shed some light on the problem by showing that the homology of $\left(\mathbb{T}^{n}\right)^{*}$ is at least as complicated as the homology of $\mathbb{T}^{n}$. We have the following more general result:

TheOREM 48. Let $X_{i}$ all be one-dimensional and put $X=X_{1} \times \ldots \times X_{n}$. Then $X$ (identified with the Dirac measures) is a retract of $X^{*}$. In particular, $H_{q}(X)$ is isomorphic to a subgroup of $H_{q}\left(X^{*}\right)$ for each $q \in \mathbb{Z}$.

Proof. Since each $X_{i}$ is one-dimensional we may identify $X_{i}$ with $X_{i}^{*}$ (cf. [14]). Let $p_{i}: X \rightarrow X_{i}$ denote the projections, $i=1, \ldots, n$. Define $P: X^{*} \rightarrow X$ by $P(\mu)=\left(\left(p_{i}\right)_{*}(\mu)\right)_{i=1}^{n}$ and let $I: X \rightarrow X^{*}$ be the inclusion map (as Dirac measures). We have

$$
(P \circ I)(x)=\left(\left(p_{i}\right)_{*}\left(\delta_{x}\right)\right)_{i=1}^{n}=\left(x_{i}\right)_{i=1}^{n}=x,
$$

i.e. $P$ is the desired retraction and the assertion follows.

6.1. Locally connected continua. In this subsection $X$ denotes a locally connected continuum. We recall a notion from [7]:

Definition 49. For a set $C \in \mathcal{C}_{\mathrm{s}}(X)$ we define the number of sides of $C$, denoted by $\operatorname{sd}(C)$, as follows:

$$
\operatorname{sd}(C)=\sup _{C \subset U \in \mathcal{O}_{\mathrm{c}}(X)} \operatorname{card}\{V: V \text { is a component of } U \backslash C\} .
$$

We denote the $n$-sided sets by $\mathcal{C}_{\mathrm{s} n} \subset \mathcal{C}_{\mathrm{s}}(X)$ for $n \in \mathbb{N} \cup\{\infty\}$, where $\mathcal{C}_{\mathrm{s} 0}=$ $\{\emptyset, X\}$.

It is shown in [7] that the genus of a space $X$ equals $\sup _{C \in \mathcal{C}_{\mathbf{s}}(X)} \operatorname{sd}(C)-1$. 
The following proposition is a straightforward generalization of Proposition 13:

Proposition 50. Let $X$ be a locally connected continuum. A map $q$ : $\mathcal{A}_{\mathrm{s}}(X) \rightarrow \mathcal{A}(Y)$ extends uniquely to an image transformation $\widetilde{q}: \mathcal{A}(X) \rightarrow$ $\mathcal{A}(Y)$ if and only if the following hold:

(a') If $A, A_{i} \in \mathcal{A}_{\mathrm{s}}$ for $i=1, \ldots, n$ and $\biguplus A_{i} \subset A$, then $\biguplus q\left(A_{i}\right) \subset q A$.

$\left(\mathrm{b}^{\prime}\right)$ If $U, U_{\lambda} \in \mathcal{O}_{\mathrm{s}}$ and $U_{\lambda} \nearrow U$ with $\lambda \in \Lambda$ where $\Lambda$ is a directed set, then $q U_{\lambda} \nearrow q U$.

$\left(\mathrm{c}^{\prime}\right)$ For any $A \in \mathcal{A}_{\mathrm{s}}$ we have $q(A) \uplus q(X \backslash A)=Y$.

$\left(\mathrm{d}^{\prime}\right)$ For any irreducible partition $\mathcal{P}$ of $X, \biguplus_{A \in \mathcal{P}} q(A)=Y$.

Proof. The proof of Proposition 13 of [4] goes through unchanged, provided we note that for genus larger than zero, $\left(\mathrm{d}^{\prime}\right)$ is necessary to ensure that the adjoint of $q$ maps Dirac measures into solid set functions (corresponding to the additional requirement (iv) for a solid set function).

7. Spaces with genus one. The property that $C_{1}, C_{2} \in \mathcal{C}_{\mathrm{s} 2}$ disjoint implies $X \backslash\left(C_{1} \cup C_{2}\right) \notin \mathcal{O}_{\text {c }}$ is shared by a large class of spaces with genus one, e.g. annuli and tori of all dimensions. This property is necessary for the results in the following propositions.

Simple topological measures on the torus have been studied in [11] and [7]. We recall some of the ideas and results from [7]:

Proposition 51. Let $X$ be a space of genus one with the property that $C_{1}, C_{2} \in \mathcal{C}_{\mathrm{s} 2}$ disjoint implies $X \backslash\left(C_{1} \cup C_{2}\right) \notin \mathcal{O}_{\mathrm{c}}$. Then there is a classification of the sets in $\mathcal{C}_{\mathrm{s}}(X)$ as follows:

(i) We have

$$
\begin{aligned}
\mathcal{C}_{\mathrm{s}}(X) & =\mathcal{C}_{\mathrm{s} 0} \uplus \mathcal{C}_{\mathrm{s} 1} \uplus \mathcal{C}_{\mathrm{s} 2}, \quad \mathcal{C}_{\mathrm{s} 1}=\mathcal{B} \uplus \mathcal{F} \uplus \mathcal{T}, \quad \mathcal{C}_{\mathrm{s} 2}=\biguplus_{S \in \mathcal{E}} S, \\
\mathcal{B} & =\left\{C \in \mathcal{C}_{\mathrm{s} 1}: \text { there is a } C^{\prime} \in \mathcal{C}_{\mathrm{s} 2} \text { with } C \cap C^{\prime}=\emptyset\right\}, \\
\mathcal{T} & =\left\{C \in \mathcal{C}_{\mathrm{s} 1}: \text { for all } \emptyset \neq C^{\prime} \subset X \backslash C, C^{\prime} \in \mathcal{B}\right\}, \\
\mathcal{F} & =\mathcal{C}_{\mathrm{s} 1} \backslash(\mathcal{B} \uplus \mathcal{T}) .
\end{aligned}
$$

(ii) Each $S \in \mathcal{E}$ is an equivalence class of the relation

$$
C \sim C^{\prime} \Leftrightarrow \exists C=C_{1}, \ldots, C_{n}=C^{\prime} \in \mathcal{C}_{\mathrm{s} 2}, C_{i} \cap C_{i+1}=\emptyset, i=1, \ldots, n-1 .
$$

(iii) If $\emptyset \neq C \subset C^{\prime} \neq X$ and $C, C^{\prime} \in \mathcal{C}_{\mathrm{s}}(X)$, then either $C \in \mathcal{B}$, or $C^{\prime} \in \mathcal{T}$, or $C, C^{\prime}$ are both in the same element of $\mathcal{E} \cup\{\mathcal{F}\}$.

(iv) If $C \cap C^{\prime}=\emptyset ; \emptyset \neq C, C^{\prime} \in \mathcal{C}_{\mathrm{s}}(X)$, then either $C \in \mathcal{B}$, or $C^{\prime} \in \mathcal{B}$, or $C, C^{\prime}$ are both in the same element of $\mathcal{E} \cup\{\mathcal{F}\}$.

(v) If $C \in \mathcal{B}$ then there is a $C^{\prime} \in \mathcal{T}$ disjoint from $C$. If $C \in \mathcal{E} \cup\{\mathcal{F}\}$ then there is a disjoint $C^{\prime}$ in the same element of $\mathcal{E} \cup\{\mathcal{F}\}$. 
(vi) If $\mathcal{P}$ is an irreducible partition of $X$, then $\mathcal{P}=\left\{C_{1}, C_{2}, U_{1}, U_{2}\right\}$ where $C_{1}, C_{2}, X \backslash U_{1}$ and $X \backslash U_{2}$ all belong to a single element of $\mathcal{E}$.

From the above description of solid sets one may construct a family of solid set functions (cf. [7]):

Proposition 52. Let $\nu$ and $\mu_{S}$ for each $S \in \mathcal{E}$ be normalized topological measures on $X$. Then the function $\tau: \mathcal{C}_{\mathrm{s}}(X) \rightarrow \mathbb{R}$ defined by

$$
\tau(C)= \begin{cases}1 & \text { if } C \in \mathcal{T}, \\ \nu(C) & \text { if } C \in \mathcal{F}, \\ \mu_{S}(C) & \text { if } C \in S \in \mathcal{E}, \\ 0 & \text { if } C \in \mathcal{B},\end{cases}
$$

is a solid set function, and hence extends uniquely to a topological measure on $X$.

We have the following basic observation:

Lemma 53. Any homeomorphism $\Phi$ preserves each of the class $\mathcal{B}, \mathcal{T}$ and $\mathcal{F}$, and induces a permutation of $\mathcal{E}$.

Proof. A homeomorphism necessarily maps solid sets to solid sets. Moreover, two-sidedness is preserved by homeomorphisms, implying that $\mathcal{B}, \mathcal{T}$ and $\mathcal{F}$ are invariant under $\Phi$. Two sets $C, C^{\prime} \in \mathcal{C}_{\text {s2 }} \backslash \mathcal{F}$ are clearly equivalent under the relation $\sim$ of Proposition 51 if and only if $\Phi(C) \sim \Phi\left(C^{\prime}\right)$, and hence $\Phi$ induces a permutation of the family $\mathcal{E}$.

Recall that an isotopy of a space $X$ is a continuous map $\Gamma: X \times[0,1] \rightarrow$ $X$ (in particular, a homotopy) such that $\Gamma(\cdot, t)$ is a homeomorphism for each $t \in[0,1]$. Two subsets $M_{1}, M_{2}$ of $X$ are ambiently isotopic if there is an isotopy $\Gamma$ of $X$ such that $\Gamma\left(M_{1}, 0\right)=M_{1}$ and $\Gamma\left(M_{1}, 1\right)=M_{2}$. (One may assume $\Gamma(\cdot, 0)$ to be the identity.) The following result will be useful in the next section:

Proposition 54. Let $X$ be a space of genus one with the property that $C_{1}, C_{2} \in \mathcal{C}_{\mathrm{s} 2}$ disjoint implies $X \backslash\left(C_{1} \cup C_{2}\right) \notin \mathcal{O}_{\mathrm{c}}$. If $C_{1}, C_{2} \in \mathcal{C}_{\mathrm{s}}$ are ambiently isotopic, then they are in the same element of $\left\{\mathcal{C}_{\mathrm{s} 0}, \mathcal{B}, \mathcal{T}, \mathcal{F}\right\} \cup \mathcal{E}$.

Proof. Let $\Gamma$ be an isotopy such that $\Gamma\left(C_{1}, 0\right)=C_{1}$ and $\Gamma\left(C_{1}, 1\right)=$ $C_{2}$. If the sets $C_{1}, C_{2}$ are not both two-sided, then the result follows from Lemma 53. Otherwise, for each $t \in[0,1]$ we have $\Gamma\left(C_{1}, t\right) \in \mathcal{C}_{\mathrm{s} 2}$; so let $K_{t} \in \mathcal{C}_{\mathrm{s} 2}$ be such that $\Gamma\left(C_{1}, t\right) \cap K_{t}=\emptyset$, and by continuity of $\Gamma$ let $\varepsilon_{t}$ be such that $\Gamma\left(C_{1}, t^{\prime}\right) \cap K_{t}=\emptyset$ for $t-\varepsilon_{t}<t^{\prime}<t+\varepsilon_{t}$. Now $\left\{\left(t-\varepsilon_{t}, t+\varepsilon_{t}\right): t \in[0,1]\right\}$ is an open covering of $[0,1]$; by compactness there are $0=t_{1}<\ldots<t_{n}=1$ such that

$$
[0,1]=\bigcup_{i=1}^{n}\left(t_{i}-\varepsilon_{t_{i}}, t_{i}+\varepsilon_{t_{i}}\right),
$$




$$
\left(t_{i}-\varepsilon_{t_{i}}, t_{i}+\varepsilon_{t_{i}}\right) \cap\left(t_{i+1}-\varepsilon_{t_{i+1}}, t_{i+1}+\varepsilon_{t_{i+1}}\right) \neq \emptyset, \quad i=1, \ldots, n-1 .
$$

Pick $t_{i+1}^{\prime}$ in the last intersection, for $i=1, \ldots, n-1$; then clearly

$$
\begin{aligned}
C_{1} & =\Gamma\left(C_{1}, t_{1}\right) \sim K_{t_{1}} \sim \Gamma\left(C_{2}, t_{2}^{\prime}\right) \sim K_{t_{2}} \sim \ldots \sim \Gamma\left(C_{n}, t_{n}^{\prime}\right) \sim K_{t_{n}} \\
& \sim \Gamma\left(C_{1}, t_{n}\right)=C_{2} .
\end{aligned}
$$

Define $\mathcal{T}^{*}=\left\{\mu \in X^{*}: \mu C=1\right.$ for all $\left.C \in \mathcal{T}\right\}$. By setting $\nu$ and $\mu_{S}$ equal to $\mu \in \mathcal{T}^{*}$ we see that $\mathcal{T}^{*}$ are exactly those members of $X^{*}$ achievable by the construction of Proposition 52 .

The image transformations were crucial tools in the preceding sections. We will present two fundamental classes of image transformations for obtaining retracts and deformation retracts in the more general setting of this section.

Proposition 55. Let $X$ be a space of genus one with the property that $C_{1}, C_{2} \in \mathcal{C}_{\mathrm{s} 2}$ disjoint implies $X \backslash\left(C_{1} \cup C_{2}\right) \notin \mathcal{O}_{\mathrm{c}}$. Define $q: \mathcal{C}_{\mathrm{s}}(X) \rightarrow \mathcal{C}(X)$ by

$$
q(C)= \begin{cases}\emptyset & \text { if } C \in \mathcal{B}, \\ C & \text { if } C \notin \mathcal{B} \cup \mathcal{T}, \\ X & \text { if } C \in \mathcal{T} .\end{cases}
$$

Then $q$ extends uniquely to an image transformation. Moreover, $q^{*}$ is a retraction of $X^{*}$ onto $\mathcal{T}^{*}$.

Proof. For $U \in \mathcal{O}_{\mathrm{s}}(X)$ we extend $q$ by $q(U)=X \backslash q(X \backslash U)$, giving $\left(\mathrm{c}^{\prime}\right)$. Conditions $\left(\mathrm{a}^{\prime}\right)$ and $\left(\mathrm{d}^{\prime}\right)$ of Proposition 50 for $q$ follow by a straightforward (but tedious) application of Proposition 51. Let $U_{\lambda} \nearrow U$ be a net of open solid sets with $U \in \mathcal{O}_{\mathrm{s}}(X)$, and put $C=X \backslash U$ and $C_{\lambda}=X \backslash U_{\lambda}, \lambda \in \Lambda$. For $C \in \mathcal{B}$ there is a $C^{\prime} \in \mathcal{T}$ disjoint from $C$, and by compactness there is a finite open cover $\left\{U_{\lambda_{i}}\right\}_{i=1}^{n}$ of $C^{\prime}$. Hence there is a $\lambda^{\prime}$ such that for $\lambda \geq \lambda^{\prime}$ we have $C_{\lambda} \in \mathcal{B}$; accordingly, $q U_{\lambda} \nearrow q U=X$. When $C \in \mathcal{T}$, we must have $C_{\lambda} \in \mathcal{T}$ for all $\lambda \in \Lambda$, yielding $q U_{\lambda}=q U=\emptyset$. If $C \in S \in \mathcal{E} \cup\{\mathcal{F}\}$, pick $C^{\prime} \in S$ with $C \cap C^{\prime}=\emptyset$. Compactness of $C^{\prime}$ yields existence of $\lambda^{\prime} \in \Lambda$ such that $\lambda \geq \lambda^{\prime}$ implies $C_{\lambda} \cap C^{\prime}=\emptyset$; hence combining (iii) and (iv) of Proposition 51 we get $C_{\lambda} \in S$, and so $q U_{\lambda}=U_{\lambda} \nearrow U=q U$. We have established all the requirements of Proposition 50; accordingly, $q$ extends uniquely to an image transformation $q: \mathcal{A}(X) \rightarrow \mathcal{A}(X)$.

Adjoints of image transformations are continuous, so $q^{*}$ maps $X^{*}$ continuously into $\mathcal{T}^{*}$. Moreover, if $\mu \in \mathcal{T}^{*}$, then $q^{*}$ will map all sets in $\mathcal{E} \cup\{\mathcal{F}\}$ to themselves and hence not change the measure of the sets. It is also clear that $q^{*} \mu$ maps $\mathcal{B}$ to zero and $\mathcal{T}$ to one. Thus $q^{*}$ restricted to $\mathcal{T}^{*}$ is the identity map.

The notion of a non-splitting topological measure is convenient when constructing image transformations (cf. [4]). A topological measure $\mu$ in $X$ is called non-splitting if it assigns non-zero measure to all open sets 
(or equivalently there are no disjoint non-empty compact sets $C, C^{\prime} \subset X$ such that $\left.\mu C+\mu C^{\prime}=\mu X\right)$. Normalized Lebesgue measure on cubes and Haar measure on the torus are natural examples of non-splitting measures. More generally all separable spaces have non-splitting measures. We have the following class of image transformations:

Proposition 56. Let $X$ be a space of genus one with the property that $C_{1}, C_{2} \in \mathcal{C}_{\mathrm{s} 2}$ disjoint implies $X \backslash\left(C_{1} \cup C_{2}\right) \notin \mathcal{O}_{\mathrm{c}}$. For a non-splitting topological measure $m$ in $X$ and a number $t \in[0,1 / 2]$ define $q_{m, t}: \mathcal{C}_{\mathrm{s}}(X) \rightarrow \mathcal{C}(X)$ by

$$
q_{m, t}(C)= \begin{cases}\emptyset & \text { if } C \in \mathcal{B} \text { and } m C<t, \\ X & \text { if } C \in \mathcal{T} \text { and } m C \geq 1-t, \\ C & \text { otherwise. }\end{cases}
$$

Then $q_{m, t}$ extends uniquely to an image transformation. Moreover, $q_{m, \text {. }}^{*}$ : $[0,1 / 2] \times X^{*} \rightarrow X^{*}$ is continuous, so that $q_{m, 1 / 2}^{*}\left(X^{*}\right)$ is a deformation retract of $X^{*}$.

Proof. For $U \in \mathcal{O}_{\mathrm{s}}(X)$ we extend $q$ by $q(U)=X \backslash q(X \backslash U)$, giving condition $\left(\mathrm{c}^{\prime}\right)$ of Proposition 50 for $q$. Notice that since $m$ is non-splitting we cannot have disjoint sets $C \in \mathcal{B}, C^{\prime} \in \mathcal{T}$ with $m C=t$ and $m C^{\prime}=1-t$; combining this with Proposition 51 one establishes conditions $\left(\mathrm{a}^{\prime}\right)$ and $\left(\mathrm{d}^{\prime}\right)$. Now assume $U_{\lambda} \nearrow U, \lambda \in \Lambda$, and again denote the complementary net by $C_{\lambda} \searrow C$. If $C \in \mathcal{E} \cup\{\mathcal{F}\}$, then the argument of Proposition 55 applies and $q C_{\lambda} \searrow q C$. If $t \leq m C<1-t$, there is a $\lambda^{\prime} \in \Lambda$ such that $\lambda \geq \lambda^{\prime}$ implies $t \leq m C_{\lambda}<1-t$ (by the regularity of $m$ ) and hence $q C_{\lambda}=C_{\lambda} \searrow C=q C$. We are left with four cases to consider. For $C \in \mathcal{T}$ and $m C<t$, we have $C_{\lambda} \in \mathcal{T}$ for all $\lambda \in \Lambda$, and hence $q C_{\lambda} \searrow q C$. If $C \in \mathcal{B}$ and $m C \geq 1-t$, pick $C^{\prime} \in \mathcal{T}$ with $C^{\prime} \cap C=\emptyset$. By compactness there is a $\lambda^{\prime} \in \Lambda$ such that $C_{\lambda^{\prime}} \cap C^{\prime}=\emptyset$ (implying that $C_{\lambda} \in \mathcal{B}$ for $\lambda \geq \lambda^{\prime}$ ), and hence $q C_{\lambda} \searrow q C$. The case $C \in \mathcal{T}$ and $m C \geq 1-t$ is trivial since it implies that $C_{\lambda} \in \mathcal{T}$ and $m C_{\lambda} \geq 1-t$ for all $\lambda \in \Lambda$. If $C \in \mathcal{B}$ and $m C<t$, there is a $\lambda^{\prime} \in \Lambda$ such that $\lambda \geq \lambda^{\prime}$ implies $m C_{\lambda}<t$ (by the regularity of $m$ ) and $C_{\lambda} \in \mathcal{B}$ (by the argument in Proposition 55), thus $q C_{\lambda}=\emptyset=q C$. Hence $q$ also satisfies ( $\left.\mathrm{b}^{\prime}\right)$ and extends uniquely to an image transformation $q: \mathcal{A}(X) \rightarrow \mathcal{A}(X)$.

Obviously $q_{m, 0}^{*}: X^{*} \rightarrow X^{*}$ is the identity map, and it is straightforward to check that $q_{m, 1 / 2}^{*}$ is idempotent. We must show that $q_{m, .}^{*}:[0,1 / 2] \times X^{*} \rightarrow$

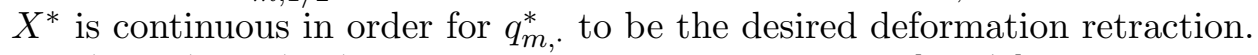
Let $\left(t_{\lambda}, \mu_{\lambda}\right) \rightarrow(t, \mu), \lambda \in \Lambda$, be a convergent net in $[0,1 / 2] \times X^{*}$. Recall that $\left\{V^{*}: V \in \mathcal{O}_{\mathrm{S}}(X)\right\}$ is a subbase for the topology of $X^{*}$, so we assume that $q_{m, t}^{*}(\mu) \in V^{*}$ for some $V \in \mathcal{O}_{\mathrm{s}}(X)$. Put $C=X \backslash V$, implying that $q_{m, t}^{*}(\mu)(C)=0$. Then we cannot have $C \in \mathcal{T}$ and $m C \geq 1-t$ by the definition of $q_{m, t}$; moreover if $C \in \mathcal{E} \cup\{\mathcal{F}\}$ then $q_{m, t_{\lambda}}(C)=q_{m, t}(C)=C$ for 
all $\lambda \in \Lambda$, trivializing that case. Now assume that $m C<t$ or $t<m C<1-t$; in both cases there is a $\lambda^{\prime}$ such that $\lambda \geq \lambda^{\prime}$ implies $m C<t_{\lambda}$ or respectively $t_{\lambda}<m C<1-t_{\lambda}$, and hence $q_{m, t_{\lambda}}(C)=q_{m, t}(C)$ (implying $q_{m, t_{\lambda}}^{*}\left(\mu_{\lambda}\right) \in V^{*}$ ). A similar argument covers the case $C \in \mathcal{T}$ and $m C=t$. Finally, assume that $m C=t$ and $C \in \mathcal{B}$. We then get $q_{m, t}(C)=C$ and accordingly $\mu(C)=0$. There is a $\lambda^{\prime} \in \Lambda$ such that $\lambda \geq \lambda^{\prime}$ implies $\mu_{\lambda}(C)=0$ and $t_{\lambda}<1-t$, thus $q_{m, t_{\lambda}}(C)=C$ or $\emptyset$ and $q_{m, t_{\lambda}}^{*}\left(\mu_{\lambda}\right) \in V^{*}$. We have established the continuity of $q_{m, \cdot}^{*}$.

Note that if two image transformations agree on solid sets they must agree on all open or closed sets by the uniqueness of their extension. We obtain the following nice property of the image transformations constructed above. The proof is straightforward from the definition in Proposition 56.

Corollary 57. Let $q_{m_{1}, t_{1}}, \ldots, q_{m_{k}, t_{k}}: \mathcal{C}(X) \rightarrow \mathcal{C}(X)$ be image transformations obtained from Proposition 56. Then

$$
q_{m_{i}, t_{i}} \circ q_{m_{j}, t_{j}}=q_{m_{j}, t_{j}} \circ q_{m_{i}, t_{i}}, \quad 1 \leq i, j \leq k .
$$

Consequently, $q_{m_{1}, 1 / 2}^{*} \circ \ldots \circ q_{m_{k}, 1 / 2}^{*}\left(X^{*}\right)$ is a deformation retract of $X^{*}$.

THEOREM 58. If $X$ admits a non-splitting probability measure $m$, then $H_{n}\left(X^{*}\right) \cong H_{n}\left(\mathcal{T}^{*}\right)$ for all $n \in \mathbb{Z}$.

Proof. Let

$$
\begin{aligned}
\mathcal{Q}=\left\{M_{\lambda}=q_{m_{1}, 1 / 2}^{*}\right. & \circ \ldots \circ q_{m_{k}, 1 / 2}^{*}\left(X^{*}\right): \\
\lambda & \left.=\left\{m_{1}, \ldots, m_{k}\right\} \text { non-splitting measures on } X\right\} .
\end{aligned}
$$

Then $\mathcal{Q}$ with inclusion maps is an inverse system of deformation retracts of $X^{*}$. Note that $H_{n}\left(q_{m_{1}, 1 / 2}^{*} \circ \ldots \circ q_{m_{k}, 1 / 2}^{*}\left(X^{*}\right)\right) \cong H_{n}\left(X^{*}\right)$ for all $n \in \mathbb{Z}$. Moreover, the inclusion maps $i: M_{\lambda_{1}} \rightarrow M_{\lambda_{2}}$ for $M_{\lambda_{1}}, M_{\lambda_{2}} \in \mathcal{Q}$ induce isomorphisms $i_{*}: H_{n}\left(M_{\lambda_{1}}\right) \rightarrow H_{n}\left(M_{\lambda_{2}}\right)$. Hence by the continuity of the Čech homology we have

$$
H_{n}\left(X^{*}\right)=\lim _{\longleftarrow} H_{n}\left(M_{\lambda}\right)=H_{n}(\bigcap \mathcal{Q}) .
$$

We have $\mathcal{T}^{*}=\bigcap \mathcal{Q}$ (completing the proof). Indeed, let $C \in \mathcal{B}$. If $m C=0$ then $q_{m, 1 / 2}(C)=\emptyset$. Since $m$ is non-splitting we must have $m C<1$, thus if $m C>0$ replace $m$ with the non-splitting measure $m^{\prime}$ given by

$$
m^{\prime}(E)=\frac{1}{3 m(C)} m(E \cap C)+\frac{2}{3(1-m C)} m(E \backslash C) .
$$

Then $q_{m^{\prime}, 1 / 2}(C)=\emptyset$, and in either case we have $\mu C=0$ for all $\mu \in \bigcap \mathcal{Q}$. Accordingly $\bigcap \mathcal{Q} \subset \mathcal{T}^{*}$; the reverse inclusion is obvious since any of the image transformations leaves $\mathcal{T}^{*}$ fixed. 
Proposition 59. Let $\pi: \prod_{S \in \mathcal{E} \cup\{\mathcal{F}\}} X^{*} \rightarrow \mathcal{T}^{*}$ be given by

$$
\pi\left(\left(\mu_{S}\right)_{S \in \mathcal{E} \cup\{\mathcal{F}\}}\right)(C)=\mu_{S}(C) \quad \text { when } C \in S .
$$

Then $\pi$ is continuous and surjective.

Proof. The map $\pi$ is well defined by Proposition 52 and surjective since $\pi((\mu))=\mu$ for $\mu \in \mathcal{T}^{*}$. Let $\left(\mu_{S}^{\lambda}\right)_{S \in \mathcal{E} \cup\{\mathcal{F}\}} \rightarrow\left(\mu_{S}\right)_{S \in \mathcal{E} \cup\{\mathcal{F}\}}$ be a convergent net in $\prod_{S \in \mathcal{E} \cup\{\mathcal{F}\}} X^{*}$. To prove the continuity it suffices to show that $\pi\left(\left(\mu_{S}^{\lambda}\right)_{S \in \mathcal{E} \cup\{\mathcal{F}\}}\right)(C)$ converges to $\pi\left(\left(\mu_{S}\right)_{S \in \mathcal{E} \cup\{\mathcal{F}\}}\right)(C)$ for $C \in \mathcal{C}_{\mathrm{s}}(X)$ when $\pi\left(\left(\mu_{S}\right)_{S \in \mathcal{E} \cup\{\mathcal{F}\}}\right)(C)=0$. This is trivially fulfilled when $C \in \mathcal{B} \cup \mathcal{T}$, so assume that $C \in S \in \mathcal{E} \cup\{\mathcal{F}\}$ and that $\pi\left(\left(\mu_{S}\right)_{S \in \mathcal{E} \cup\{\mathcal{F}\}}\right)(C)=\mu_{S}(C)=0$. By the properties of the product topology, $\mu_{S}^{\lambda} \rightarrow \mu_{S}$ for each $S \in \mathcal{E} \cup\{\mathcal{F}\}$ and thus $\pi\left(\left(\mu_{S}^{\lambda}\right)_{S \in \mathcal{E} \cup\{\mathcal{F}\}}\right)(C)=\mu_{S}^{\lambda}(C) \rightarrow 0=\mu_{S}(C)$.

The map $\pi$ above is not injective, but this can be achieved if we choose the product space more restrictively. We have the following useful result:

Theorem 60. For $\mu_{0} \in \mathcal{T}^{*}$ put

$$
\mathcal{M}_{S}^{\mu_{0}}=\left\{\mu \in \mathcal{T}^{*}: \mu C=\mu_{0}(C), C \in \mathcal{C}_{\mathrm{s}} \backslash S\right\}, \quad S \in \mathcal{E} \cup\{\mathcal{F}\} .
$$

Then the restriction $\pi: \prod_{S \in \mathcal{E} \cup\{\mathcal{F}\}} \mathcal{M}_{S}^{\mu_{0}} \rightarrow \mathcal{T}^{*}$ is a homeomorphism.

Proof. By Proposition 59 it suffices to show that $\pi$ is bijective and that each $\mathcal{M}_{S}^{\mu_{0}}$ is compact. For the latter let $\mu_{\lambda} \rightarrow \mu$ be a convergent net in $\mathcal{M}_{S}^{\mu_{0}}$. By Proposition $55, \mathcal{T}^{*}$ is compact, so we must have $\mu \in \mathcal{T}^{*}$. If $C \notin S$ and $\mu_{0}(C)=0$, pick an open solid set $U$ such that $C \subset U, \mu_{0}(U)=0$ (by regularity of $\mu_{0}$ ) and $X \backslash U \notin S$ (by Proposition 51(iii), (iv) and (v)). Then $\mu_{\lambda}(U)=0$ for all $\lambda \in \Lambda$, and thus $\mu U=0$, implying $\mu C=0$. Conversely, $\mu C=0$ and $C \notin S$ implies $\mu_{0}(C)=\mu_{\lambda}(C) \searrow 0$, so $\mu_{0}(C)=0$. Thus $\mu \in \mathcal{M}_{S}^{\mu_{0}}$, establishing compactness of $\mathcal{M}_{S}^{\mu_{0}}$.

For $\mu \in \mathcal{T}^{*}$ let $\mu_{S}$ be given, as in Proposition 52, by

$$
\mu_{S}(C)=\left\{\begin{array}{ll}
\mu_{0}(C), & C \notin S, \\
\mu C, & C \in S,
\end{array} \quad C \in \mathcal{C}_{\mathrm{s}} .\right.
$$

Then $\mu_{S} \in \mathcal{M}_{S}^{\mu_{0}}$ and $\pi\left(\left(\mu_{S}\right)_{S \in \mathcal{E} \cup\{\mathcal{F}\}}\right)=\mu$, so $\pi$ is surjective.

Assume $\left(\mu_{S}\right)_{S \in \mathcal{E} \cup\{\mathcal{F}\}} \neq\left(\mu_{S}^{\prime}\right)_{S \in \mathcal{E} \cup\{\mathcal{F}\}} \in \prod_{S \in \mathcal{E} \cup\{\mathcal{F}\}} \mathcal{M}_{S}^{\mu_{0}}$. Then for some $C \in S \in \mathcal{E} \cup\{\mathcal{F}\}$ we must have $\mu_{S}(C) \neq \mu_{S}^{\prime}(C)$, implying $\pi\left(\left(\mu_{S}\right)_{S \in \mathcal{E} \cup\{\mathcal{F}\}}\right)(C)$ $\neq \pi\left(\left(\mu_{S}^{\prime}\right)_{S \in \mathcal{E} \cup\{\mathcal{F}\}}\right)(C)$, so $\pi$ is injective.

8. The torus. We will now apply the results of the previous section to investigate the topological measures on the torus. Before we can embark on the homological results we need to work out the basic properties of compact solid sets in the torus. Throughout the section we will let $\mathbb{T}^{2}$ denote the torus. 
Consider the maps

$$
\phi^{(p, q)}: \mathbb{T} \rightarrow \mathbb{T}^{2} \quad \text { defined by } \quad \phi^{(p, q)}(z)=\left(z^{p}, z^{q}\right), p, q \in \mathbb{Z} .
$$

The family $\left\{\phi^{(p, q)}\right\}_{p, q \in \mathbb{Z}}$ with coordinatewise multiplication is isomorphic to the fundamental group $\pi\left(\mathbb{T}^{2}\right)$ of the torus. Of course, the torus itself also has a group structure as a Lie group. We recall the following:

Lemma 61. Let $p_{1}, q_{1}, p_{2}, q_{2} \in \mathbb{Z}, p_{1} q_{2}-p_{2} q_{1}=1$. Then the map $\Phi$ : $\mathbb{T}^{2} \rightarrow \mathbb{T}^{2}$ given by

$$
\Phi\left(z_{1}, z_{2}\right)=\phi^{\left(p_{1}, q_{1}\right)}\left(z_{1}\right) \phi^{\left(p_{2}, q_{2}\right)}\left(z_{2}\right)=\left(z_{1}^{p_{1}} z_{2}^{p_{2}}, z_{1}^{q_{1}} z_{2}^{q_{2}}\right)
$$

is an automorphism of $\mathbb{T}^{2}$. In particular, $\Phi$ is a homeomorphism and maps line segments to line segments.

REMARK 62. Note that the map $\Phi$ induces a change of basis in the fundamental group $\pi\left(\mathbb{T}^{2}\right)$.

We need to assign curves to each of the classes of solid sets in Proposition 51. This is achieved by the following proposition:

Proposition 63. Let $C \in S \in \mathcal{E} \cup\{\mathcal{F}, \mathcal{B}, \mathcal{T}\}$ and $C \subset U \in \mathcal{O}$. Then there is a solid set $K \in S$ such that $C \subset K^{\circ} \subset K \subset U$ and the boundary of $K$ consists of $\operatorname{sd}(C)$ polygons which are simple closed curves.

Proof. Let $C \in S \in \mathcal{E} \cup\{\mathcal{F}\}$ be arbitrary, pick a disjoint set $C^{\prime} \in S$, and pick an open set $U^{\prime}$ with $C \subset U^{\prime} \subset X \backslash C^{\prime} \cup U$ and $X \backslash U^{\prime} \in \mathcal{C}_{\text {c }}$. Equip $\mathbb{T}^{2}$ with a metric $d$ and put $r=\frac{1}{2} \min \left\{d\left(C, X \backslash U^{\prime}\right)\right\}$; further, let $\varepsilon<r / 2$ and let $D_{l, k}, 0 \leq l, k \leq(1-\varepsilon) /(r-\varepsilon), l, k \in \mathbb{N}$, denote the overlapping squares

$$
D_{l, k}=\left\{\left(e^{2 \pi \theta i}, e^{2 \pi \phi i}\right) \in \mathbb{T}^{2}: l \leq \frac{\theta}{r-\varepsilon} \leq r+l, k \leq \frac{\phi}{r-\varepsilon} \leq r+k\right\} .
$$

Then there is an index set $I$ such that $\left\{D_{l, k}^{\circ}\right\}_{(l, k) \in I} \operatorname{covers} C$ and $\mathbb{T}^{2} \backslash C^{\prime} \supset$ $\bigcup_{I} D_{l, k} \in \mathcal{C}_{\mathrm{s}}$. Now exactly one of the co-components of $\bigcup_{I} D_{l, k}$ contains $X \backslash U^{\prime}$ (and consequently $C^{\prime}$ ) by connectedness of $X \backslash U^{\prime}$. Adding the squares covering the other co-components of $\bigcup_{I} D_{l, k}$ to $\bigcup_{I} D_{l, k}$ we conclude that $C \sim \bigcup_{I} D_{l, k}$.

It is clear that the boundary of $\bigcup_{I} D_{l, k}$ consists of polygons. It is also clear from the construction that these polygons will be simple closed curves. By the definition of sides there is an open set $O$ (we may assume $O \cap C^{\prime}=\emptyset$ ) such that $O \backslash C$ has two components covering the polygons, implying at least two polygons; conversely, more than two components in the boundary would imply that $C$ has more than two sides.

A similar argument yields the desired result for $C \in \mathcal{B}$ and for $C \in \mathcal{T}$.

Corollary 64. Let $C \in S \in \mathcal{E}$. The procedure in Proposition 63 will produce a boundary consisting of two polygons, each equivalent to $C$. 
Proof. The boundary of $K$ will consist of two polygons, say $l_{1}$ and $l_{2}$, since $C \in \mathcal{C}_{\mathrm{s} 2}$. Each polygon is solid, e.g. $l_{1}$ is solid since $\mathbb{T}^{2} \backslash l_{1}=\left(\bigcup_{I} D_{l, k}^{\circ}\right) \cup$ $l_{2} \cup\left(\mathbb{T}^{2} \backslash \bigcup_{I} D_{l, k}\right)$ and $l_{2}$ is in the closure of both $\bigcup_{I} D_{l, k}^{\circ}$ and $\mathbb{T}^{2} \backslash \bigcup_{I} D_{l, k}$.

Each polygon is two-sided since $l_{2}^{\mathrm{c}} \backslash l_{1}$ and $l_{1}^{\mathrm{c}} l_{2}$ have two components each. Both polygons are disjoint from $C$ and two-sided, hence equivalent to $C$.

We include a proof of the following for the reader's convenience.

Lemma 65. Let $\phi$ be a piecewise linear, simple closed curve in $\mathbb{T}^{2}$. Then $\phi(\mathbb{T})$ is ambiently isotopic either to $\phi^{(p, q)}(\mathbb{T})$ for some $p, q \in \mathbb{Z}$ with $p \geq 0$ and $\operatorname{gcd}(p, q)=1$, or to an arbitrarily small square boundary.

Proof. By Lemma 61 we may assume that $\phi$ is homotopic to $\phi^{(n, 0)}$ for some $n \in \mathbb{N}$.

There is an ambient isotopy mapping each line segment of $\phi$ to a piecewise linear function with vertical and horizontal line segments. Indeed, let $l$ be a line segment in $\phi$, and let $l_{1}, l_{2}$ denote the adjacent line segments. Put $r=d\left(l, \phi(\mathbb{T}) \backslash\left\{l, l_{1}, l_{2}\right\}\right)$. Now pick a curve, say $\phi_{1}$, connecting the endpoints of $l$ such that each step has height and length smaller than $r$, intersecting $l$ in each step. Further, $\phi_{1}$ shall have a finite number of steps and be disjoint from $l_{1}$ and $l_{2}$ (see Figure 1).

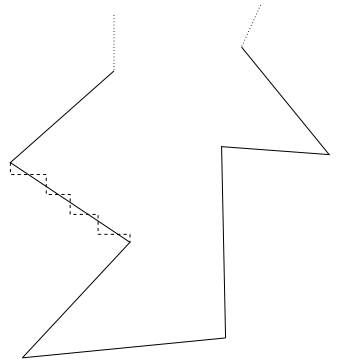

Fig. 1

The curve $\phi_{1}$ is then disjoint from $\phi(\mathbb{T}) \backslash\{l\}$, so there exists an isotopy leaving $\phi(\mathbb{T}) \backslash\{l\}$ invariant and with $l$ ambiently isotopic to $\phi_{1}$ (the isotopy may be constructed locally for each step). This procedure is carried out for each line segment of $\phi$, replacing the previous curve with a new ambiently isotopic curve in each step. Composing the isotopies we have $\phi(\mathbb{T})$ ambiently isotopic to a piecewise linear curve, say $\phi^{\prime}$, with vertical and horizontal line segments.

The next step is to "straighten out" $\sqsupset$ shaped pieces of $\phi^{\prime}$, i.e. where $\phi^{\prime}$ makes the same turn twice. Start where the distance between the turns is minimal (see Figure 2).

Then the rectangle (with interior) in Figure 2 is disjoint from the rest of $\phi^{\prime}$. Thus there is an isotopy leaving the remainder of $\phi^{\prime}$ invariant such that the $\sqsupset$ shaped piece is ambiently isotopic to a straight line. Again repeating 


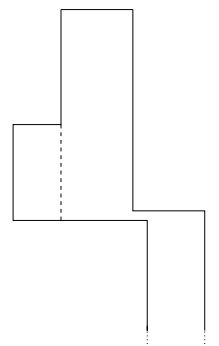

Fig. 2

the process inductively on the new curve, and then composing the isotopies, we get a curve ambiently isotopic to $\phi^{\prime}$. Note that each step in the induction reduces the number of line segments in $\phi^{\prime}$, so the induction must stop. The new curve has two possibilities:

If all the horizontal (and vertical) line segments have the same direction, then since $\phi$ is homotopic to $\phi^{(n, 0)}$ the curve must be a straight line, and consequently $n=1$.

If the curve has horizontal (or vertical) segments with opposite directions, then it must be a square. In that case we get $n=0$, and $\phi(\mathbb{T})$ is ambiently isotopic to an arbitrarily small square boundary.

TheOREM 66. We have $\mathcal{F}=\emptyset$, and there is one class $S_{(p, q)} \in \mathcal{E}$ for each $(p, q)$ with $p, q \in \mathbb{Z}, p \geq 0$ and $\operatorname{gcd}(p, q)=1$. Suitable representatives are given by the curves $\left\{\phi^{(p, q)}\right\}$ (presented at the beginning of this section).

Proof. Assume $C \in S \in\{\mathcal{F}\} \cup \mathcal{E}$, and apply Proposition 63 to obtain a solid set $K \in S$ such that $C \subset K^{\circ} \subset K$ and the boundary of $K$ consists of $\operatorname{sd}(C)$ polygons which are simple closed curves. Let $l$ denote one of the polygons in the boundary of $K$. Consider Lemma 65. If $K \in \mathcal{F}$ then $l$ is not solid since $X \backslash l=K^{\circ} \uplus X \backslash K$, and so must be ambiently isotopic to an arbitrarily small square boundary. Hence the isotopy from Lemma 65 must transfer $K$ either to the square or the exterior of the square, neither of which is in $\mathcal{F}$, a contradiction. Accordingly $\mathcal{F}=\emptyset$.

Now assume that $K \in S \in \mathcal{E}$. Then $l \in S$ by Corollary 64, in particular $l$ is solid and so ambiently isotopic to $\phi^{(p, q)}(\mathbb{T})$ for some $p, q \in \mathbb{Z}$ with $p \geq 0$ and $\operatorname{gcd}(p, q)=1$. Consequently, $\phi^{(p, q)}(\mathbb{T}) \in S$.

We have shown that each class $S \in \mathcal{E}$ contains at least one curve $\phi^{(p, q)}(\mathbb{T})$ and clearly each such curve is a member of exactly one $S \in \mathcal{E}$, giving a well defined surjective map from $\left\{\phi^{(p, q)}\right\}$ to $\mathcal{E}$. It remains to show injectivity:

If $\phi^{(p, q)} \in S$, then $\phi^{(p, q)}$ is ambiently isotopic to any piecewise linear simple closed curve in $S$ by Lemma 65 (the existence of such curves in $S$ is ensured by Proposition 63). In particular, if $\phi^{(p, q)}, \phi^{\left(p^{\prime}, q^{\prime}\right)} \in S$, then $\phi^{(p, q)}$ and $\phi^{\left(p^{\prime}, q^{\prime}\right)}$ must be homotopic, implying $p=p^{\prime}$ and $q=q^{\prime}$. 
Similarly to Theorem 60 , for any $\mu_{0} \in \mathcal{T}^{*}$ and $S \in \mathcal{E}$ we define a map $\pi_{S}^{\mu_{0}}:\left(\mathbb{T}^{2}\right)^{*} \rightarrow \mathcal{M}_{S}^{\mu_{0}}$ by $\mu \mapsto \mu_{S}$ with

$$
\mu_{S}(C)=\left\{\begin{array}{ll}
\mu_{0}(C), & C \notin S, \\
\mu C, & C \in S,
\end{array} \quad C \in \mathcal{C}_{\mathrm{s}} .\right.
$$

Then $\pi_{S}^{\mu_{0}}$ is continuous. Indeed, let $\mu_{\lambda} \rightarrow \mu$ be a convergent net in $\left(\mathbb{T}^{2}\right)^{*}$. Analogously to previous arguments it is straightforward to verify that $\mu_{S}(C)$ $=0$ implies $\mu_{\lambda S}(C) \rightarrow 0$.

We have the following:

Lemma 67. For $\mu_{0} \in\left(\mathbb{T}^{2}\right)^{*}, \mathcal{M}_{S}^{\mu_{0}}$ from Theorem 60 has a retract homeomorphic to the circle.

Proof. By Theorem 66 let $S=S_{(p, q)}$; we may assume by Lemmas 53 and 61 that $(p, q)=(1,0)$. Take $P: \mathbb{T}^{2} \rightarrow \mathbb{T}$ to be the projection $\left(z_{1}, z_{2}\right) \mapsto z_{2}$. We have $P \circ \phi^{(0,1)}=\mathrm{id}_{\mathbb{T}}$, so $\phi^{(0,1)} \circ P$ is a retraction of $\mathbb{T}^{2}$ onto $\phi^{(0,1)}(\mathbb{T}) \cong \mathbb{T}$. We proceed to show that $\left.\pi_{S}^{\mu_{0}} \circ \phi_{*}^{(0,1)} \circ P_{*}\right|_{\mathcal{M}_{S}^{\mu_{0}}}$ is our desired retraction:

Consider the continuous map $P_{*} \circ \pi_{S}^{\mu_{0}} \circ \phi_{*}^{(0,1)}: \mathbb{T}^{*} \rightarrow \mathbb{T}^{*}$. It suffices to show that $P_{*} \circ \pi_{S}^{\mu_{0}} \circ \phi_{*}^{(0,1)}$ is the identity on $\mathbb{T}^{*}$. Note that $\mathbb{T}^{*}=\left\{\delta_{z}: z \in \mathbb{T}\right\}$ since $\mathbb{T}$ is one-dimensional, so let $z \in \mathbb{T}$ be arbitrary. Then $P^{-1}(z) \in S$ and

$$
\begin{aligned}
\left(P_{*} \circ \pi_{S}^{\mu_{0}} \circ \phi_{*}^{(0,1)}\right)\left(\delta_{z}\right)(\{z\}) & =\left(\pi_{S}^{\mu_{0}} \circ \phi_{*}^{(0,1)}\right)\left(\delta_{z}\right)\left(P^{-1}(z)\right)=\phi_{*}^{(0,1)}\left(\delta_{z}\right)\left(P^{-1}(z)\right) \\
& =\delta_{z}\left(\left(\phi^{(0,1)}\right)^{-1}\left(P^{-1}(z)\right)\right)=\delta_{z}(\{z\})=1,
\end{aligned}
$$

i.e. $\left(P_{*} \circ \pi_{S}^{\mu_{0}} \circ \phi_{*}^{(0,1)}\right)\left(\delta_{z}\right)=\delta_{z}$, and $P_{*} \circ \pi_{S}^{\mu_{0}} \circ \phi_{*}^{(0,1)}=\mathrm{id}_{\mathbb{T}^{*}}$.

Theorem 68 . Let $X=\mathbb{T}^{2}$. Then $X^{*}$ has tori of arbitrary countable dimensions as retracts. In particular, $H_{n}\left(X^{*}\right)$ is of infinite rank for all $n \in \mathbb{N}$.

Proof. Let $n \in \mathbb{N}$ and $\mu_{0} \in \mathcal{T}^{*}$. From Proposition 55 we know that $\mathcal{T}^{*}$ is a retract of $X^{*}$. By Proposition 60 we have $\prod_{S \in \mathcal{E}} \mathcal{M}_{S}^{\mu_{0}} \cong \mathcal{T}^{*}$, and by Lemma 67 we may retract $n$ of the countably many factors $\mathcal{M}_{S}^{\mu_{0}}$ down to a circle. The remaining factors of $\prod_{S \in \mathcal{E}} \mathcal{M}_{S}^{\mu_{0}}$ may be retracted to single point sets. The resulting space $\mathbb{T}^{n}$ is hence a retract of $X^{*}$. We may retract all factors to a circle, obtaining $\mathbb{T}^{\mathbb{N}}$ as a retract of $X^{*}$, and hence $H_{n}\left(X^{*}\right)$ is of infinite rank for all $n \in \mathbb{N}$.

\section{References}

[1] J. F. Aarnes, Quasi-states and quasi-measures, Adv. Math. 86 (1991), 41-67.

[2] -, Pure quasi-states and extremal quasi-measures, Math. Ann. 295 (1993), 575-588.

[3] -, Construction of non-subadditive measures and discretization of Borel measures, Fund. Math. 147 (1995), 213-237.

[4] J. F. Aarnes, Ø. Johansen and A. B. Rustad, Topological measures, image transformations and self-similar sets, submitted. 
[5] F. E. Browder, On the fixed point index for continuous mappings on locally connected spaces, Summa Brasil. Math. 4 (1960), 253-293.

[6] S. Eilenberg and N. Steenrod, Foundations of Algebraic Topology, Princeton Univ. Press, 1952.

[7] D. J. Grubb, Irreducible partitions and the construction of quasi-measures, Trans. Amer. Math. Soc. 353 (2001), 2059-2072.

[8] D. Grubb and T. LaBerge, Spaces of quasi-measures, Canad. Math. Bull. 42 (1999), 291-297.

[9] —, - Additivity of quasi-measures, Proc. Amer. Math. Soc. 126 (1998), 3007-3012.

[10] Ø. Johansen and A. B. Rustad, Constructing topological measures and image transformations by Boolean functions, submitted.

[11] F. F. Knudsen, Topology and the construction of extreme quasi-measures, Adv. Math. 120 (1996), 302-321.

[12] J. van Mill, Supercompactness and Wallman Spaces, Math. Centre Tracts 85, Matematisch Centrum, Amsterdam, 1977.

[13] A. B. Rustad, The multidimensional median and sample median defined as topological measures, submitted.

[14] R. Wheeler, Quasi-measures and dimension theory, Topology Appl. 66 (1995), 7592.

Department of Mathematical Sciences

Norwegian University of Science and Technology

N-7491 Trondheim, Norway

E-mail: oerjan@nvg.ntnu.no

alfr@math.ntnu.no

Received 12 March 2002;

in revised form 15 November 2002 